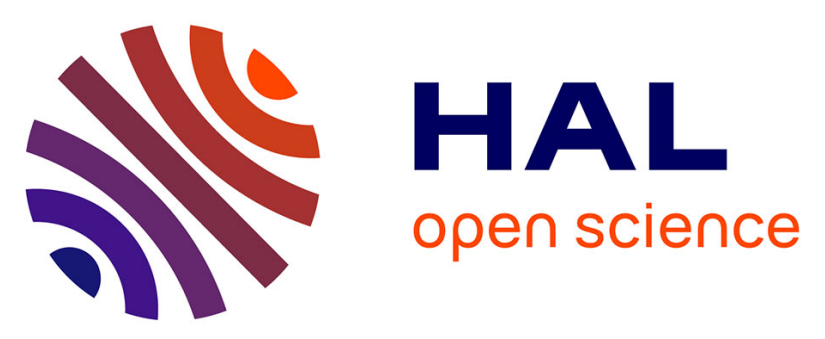

\title{
Electronically Rewritable Chipless RFID Tags Fabricated Through Thermal Transfer Printing on Flexible PET Substrates
}

M P Jayakrishnan, Sergio Lopez-Soriano, Arnaud Vena, Brice Sorli, Ira Susanti, Etienne Perret

\section{To cite this version:}

M P Jayakrishnan, Sergio Lopez-Soriano, Arnaud Vena, Brice Sorli, Ira Susanti, et al.. Electronically Rewritable Chipless RFID Tags Fabricated Through Thermal Transfer Printing on Flexible PET Substrates. IEEE Transactions on Antennas and Propagation, 2021, 69 (4), pp.1908-1921. 10.1109/TAP.2020.3030965 . hal-03235826

\section{HAL Id: hal-03235826 \\ https://hal.science/hal-03235826}

Submitted on 26 May 2021

HAL is a multi-disciplinary open access archive for the deposit and dissemination of scientific research documents, whether they are published or not. The documents may come from teaching and research institutions in France or abroad, or from public or private research centers.
L'archive ouverte pluridisciplinaire HAL, est destinée au dépôt et à la diffusion de documents scientifiques de niveau recherche, publiés ou non, émanant des établissements d'enseignement et de recherche français ou étrangers, des laboratoires publics ou privés. 


\title{
Electronically Rewritable Chipless RFID Tags Fabricated Through Thermal Transfer Printing on Flexible PET Substrates
}

\author{
M. P. Jayakrishnan, Sergio Lopez-Soriano, Arnaud Vena, Brice Sorli, Ira Susanti, and Etienne Perret, \\ Senior Member, IEEE
}

\begin{abstract}
We present an electronically rewritable chipless RFID tag based on integrated non-volatile CBRAM RF switches. Conductive Bridging Random Access Memory (CBRAM) is a non-volatile memory technology, which is identified as an innovative RF switching solution in this decade. Reconfigurable resonator or RF encoding particle (REP) used in this tag is a closed loop resonator integrated with two switches each to tune its electrical length, and encodes 3 states each, to represent more than one bit per REP. Presented tag is composed of three such REPs to represent a total of 27 unique states. This chipless RFID tag with CBRAM switches could be written/rewritten electronically using DC voltage pulses, to encode a desired code from a given combination. Thermal impression transfer printing is utilized for printing metallic layers of the presented chipless tag. Complete fabrication steps of presented tag with integrated CBRAM switches are carried out without any 'clean room' processes. An insight to working mechanism of presented rewritable REPs using electrical models is included in this paper. Proof of concept of a potential hybrid data encoding technique combining frequency shift coding and RCS magnitude level coding, through experimental studies, for presented REPs, is also given herewith.

Index Terms - Chipless RFID, Conductive Bridging Random Access Memory (CBRAM), Electronically Rewritable Chipless RFID, Metal Insulator Metal (MIM) Switches, Printing, Rewritable Chipless RFID, Thermal Transfer Printing
\end{abstract}

\section{INTRODUCTION}

Chipless RFID technology using multiple RF scatterers is a recent innovation in the RFID mainstream. Generally a

This project has received funding from the European Research Council (ERC) under European Union's Horizon 2020 research and innovation program (grant agreement No 772539, ScattererID project) https://www.scattererid.eu/. (Corresponding author: M. P. Jayakrishnan)

Jayakrishnan M.P. and Etienne Perret are with the Université Grenoble Alpes, Grenoble INP, LCIS, 26000 Valence, France. Etienne Perret is also a member of Institut Universitaire de France, 75005 Paris, France. Email: jayakrishnan.mp@1cis.grenoble-inp.fr, etienne.perret@lcis.grenoble-inp.fr

Arnaud Vena, and Brice Sorli are with the Institut d'Electronique et des Systèmes, Université de Montpellier / CNRS, Montpellier 34095, France. Email: arnaud.vena@umontpellier.fr, brice.sorli@ies.univ-montp2.fr

Sergio Lopez-Soriano was formerly with the Université Grenoble Alpes, Grenoble INP, LCIS, 26000 Valence, France, and now with the Department of Communications and Information Technology, Universitat Pompeu Fabra, Barcelona, Spain. Email: sergio.lopez@upf.edu

Ira Susanti is with the Faculty of Mathematics and Natural Science, Bandung Institute of Technology, Bandung, Indonesia. (Formerly with Université de Montpellier, France.) Email: irasusanti.itb@gmail.com multiple scatterer based chipless RFID tag is composed of a group of narrow band metallic resonators (also known as RFencoding particles or REP). These REPs function at the same time as data encoding scheme and also as receiving and backscattering antennas [1]-[5]. Data is encoded in these REPs in form of narrow band resonances, or group-delay variations, or phase encryption, at certain predefined values, in a given bandwidth, subdivided into certain windows to represent the bit pattern [2], [6], [7]. Chipless RFID tags based on multi-scatter REPs are often referred to as RF barcodes [4], [8], due to their similarity in appearance, and operation, in comparison to optical barcode technology, even though the operating wavelength and data encoding techniques are different.

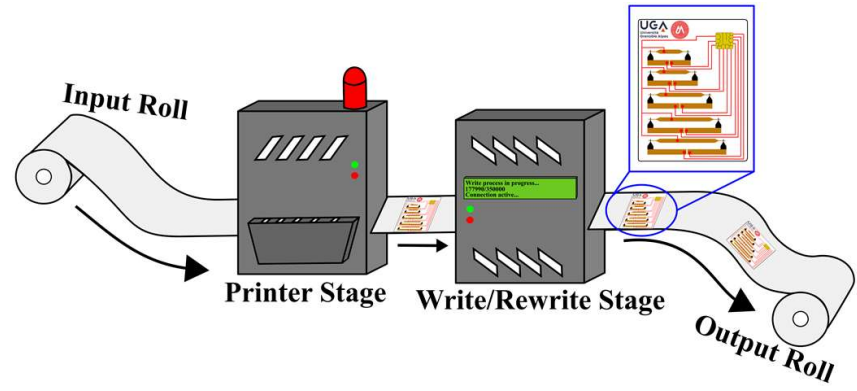

Fig. 1. Roll to roll process for manufacture of electronically rewritable chipless RFID tags, similar to as reported in this article. Write/rewrite stage maybe excluded to manufacture blank tags, which could be field programmed by a customer at the time of application.

Chipless RFID technology has much more to offer in contrast to optical barcode system, including features like non optical line of sight reading, sensor integration capability, electronic rewriteablity and so on [9]-[12].

Electronic rewriteablity gives a great deal of advantages to chipless RFID including, manufacturing cost reduction enabling fabrication of blank tags in bulk, tag reuse, ease of application as each tag could be field programmed, and so on. However, until now this functionality is not yet demonstrated in an inventive approach compatible with mass production, and hence is a primary objective of this article. With such an innovation, one could fill the technology gap among chipless RFID and conventional RFID with semiconductor chip, to some extent as shown in Fig. 1. As indicated above, in Fig. 1 a genre of chipless RFID tags could be manufactured as a single copy tag, through a roll to roll process and then either 
written/rewritten using a similar process, or retained as a raw tag to be later written or rewritten by a customer using a mobile programmer (similar to a credit card reader), at the time of application.

Electronic rewriteablity of multiple REP based chipless RFID tags primarily depends on modifying the encoding parameter (resonance frequency, phase, or group delay) of each REPs. Among these, the mostly utilized parameter is resonance frequency captured in form of Radar Cross Section (RCS), due to its simplicity in detection and stability with different detection platforms and reading distance, with minimum pre-calibration. In addition, RCS response of a particular tag captured using any reader system with a desired accuracy is expected to produce a unique response. Resonance frequency of a REP could be tuned by modifying the resonant electrical length, and this is one of the most popular data encoding strategies reported [1]-[3], [5], [13].

Many articles on reconfigurable chipless RFID describes the use of several techniques like inkjet printing, laser cutting, micromachining etc., to tune the electrical length of a REP to achieve a desired frequency of resonance [2], [6], [14], [15]. However, most of these techniques are only irreversible mechanical configurations, or write only mechanism.

As an improvement to this, recently we have reported the usage of solid state non-volatile CBRAM switching technology [21], [22] for fully passive electronically rewritable chipless RFID tags [11], [16] with simple fabrication techniques. These works are a proof of concept of application of non-volatile CBRAM switches for fully passive electronically reconfigurable REPs for chipless RFID tags on rigid/flexible substrates.

In this article for the first time, we present the design, fabrication, and experimental validation of fully passive fully printed electronically rewritable chipless RFID tags based on non-volatile CBRAM RF switching technology on flexible Polyethylene terephthalate (PET) substrates. These tags are composed of rewritable loop resonators that encodes more than one bit per REP, in contrast to previous realizations. We introduce a new additive manufacturing technique based on thermal transfer printing (compatible with roll to roll industrial processes for mass production), for realization of electronically rewritable chipless RFID tags with integrated CBRAM switches, on flexible substrates, and notably without involving any 'clean room' techniques.

In addition an investigation on the concept of a novel hybrid data encoding technique based on filament resistance control of integrated CBRAM switches is presented through experimental results. We also present detailed analysis and discussions, related to techniques, material optimization, and characteristics of presented devices.

A notable difference with presented approach in comparison to classic techniques of mechanical/printed irreversible configurability, other than the rapid realization through proposed printing technique compatible with roll to roll process, is as follows.

In classic approaches of chipless RFID tags as indicated above, each REP of definite geometry present on a tag is capable of representing only one state or code. With this approach it is very tedious and time consuming to optimize or realize a tag for a given code (in a group of possible combinations) through full wave electromagnetic simulations. Electromagnetic simulations of this sort for each and every code in a given group (even if the desired code length is not so large $\sim 10$ bits), should take care of all possible bottlenecked challenges as explained. These include, coupling between REPs, optimizing a desired resonance in a given frequency margin taking into consideration all related fabrication tolerances, maintaining the desired quality factor, and similar. This is for all combinations (1024 runs for 10 bits), and could be exhaustingly time and resource consuming. It means that most of the tags would be realized without any full wave electromagnetic simulations.

However, an evident advantage with the presented technique of electronically rewritable REPs with switches is that, each REP represents more than one code and could be operated electronically. In addition, the electromagnetic (RCS) response of all possible combinations of a tag (with a given number of REPs) could be computed analytically or numerically. This is by using a simple electrical model, similar to as presented in the following section III.B of this article. This could lead to fast and efficient design optimizations, saving ample time invested on full wave electromagnetic simulations.

\section{CBRAM SWITCH TECHNOLOGY}

CBRAM is a non-volatile memory technology reported around 1996 from the Arizona State University and was initially called a Programmable Metallization Cell (PMC) [17]. CBRAM was initially proposed as a non-volatile memory technology, which is so competent to be introduced as a commercial memory [18] and technically proficient enough to invite a prestigious DARPA grant [19] of the US government. In addition, it was later identified as a suitable candidate to be an efficient nano-metric non-volatile RF switch [20]-[23].

In construction, a basic CBRAM cell is a sandwich structure in which two electrochemically asymmetric metallic electrodes interpose a 'fast ion conductor' layer (which is also an electronic insulator) as shown in Fig. 2. This forms a structure very similar to a parallel plate capacitor.

Here, one of the electrodes is an electrochemically active metal, which could be easily oxidized on application of an electric field, to liberate positive ions, like silver and copper. The other electrode is a relatively electrochemically inert ionacceptor like aluminum, gold, platinum, tungsten, or similar [17], [24]. Suitable materials for ion conductor application include, oxidizable metal doped chalcogenide glass [17], [21], ion conducting and metal doped polymers [25]-[27], semiconductor oxides [23], [28], and even nano metrically spaced air gaps [22]. CBRAM switches are also called MetalInsulator-Metal (MIM) switches with reference to their layer structure.

Working principle of a CBRAM cell could be explained as follows with tight reference to the structure shown in Fig. 2. 
Initially, resistance across CBRAM cell is very high due to the presence of ion conductor layer, which is also an electronic insulator. However, a sufficiently high electric field applied to the CBRAM cell, which is positive with respect to the active electrode would initiate a redox mechanism. Upon this the active electrode is oxidized to liberate metallic ions. These ions move through the ion conductor under influence of applied electric field to reach the inert electrode and to get reduced to metallic atoms, and attach there.

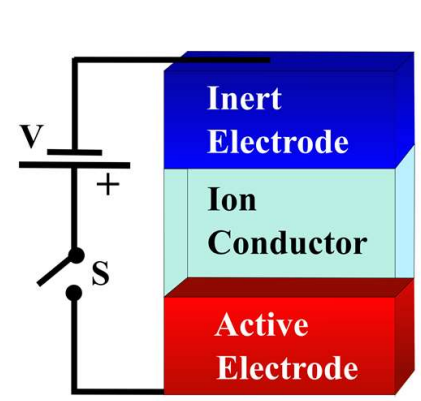

(a)

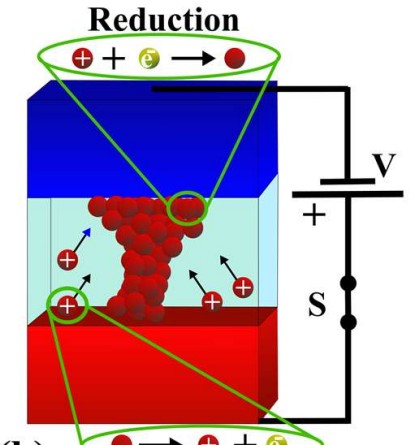

(b)
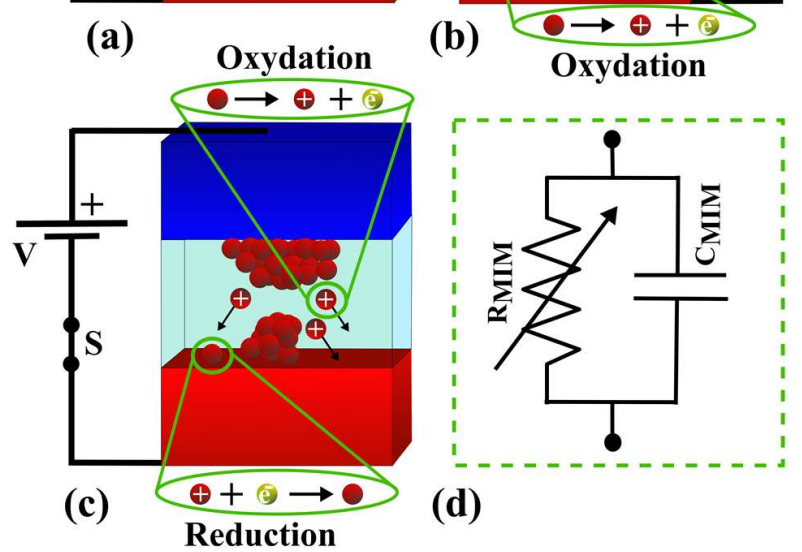

(d)

Fig. 2. Basic layer architecture and working principle of CBRAM switches (a) CBRAM switch-cell structure. (b) Set (ON) process. (c) Reset (OFF) process. (d) Electrical model.

This would lead to the formation of a dendrite shaped metallic filament from inert electrode towards the active electrode, as shown in Fig. 3 (Fig. 3 is an outcome of a control experiment, using Nafion polymer as ion conductor, to observe filament formations in copper/Nafion/aluminum CBRAM cells [29]). With prolonged exposure of the cell to above mentioned electric field, the metallic filament touches the active electrode, thereby reducing net resistance across CBRAM cell and establishing set (ON) state as shown in Fig. 2 (b) [28].

Conversely, an electric field of sufficient amplitude and opposite polarity with respect to the set $(\mathrm{ON})$ state would reverse the redox mechanism direction. This would lead to oxidation of the metallic filament attached to inert electrode, leading to liberation of ions that move towards the active electrode and get reduced there. Resulting in rupture of the metallic filament and considerably increasing impedance across the cell leading to establishment of reset (OFF) state as shown in Fig. 2 (c) [28]. A prolonged exposure of the cell to reset electric field would lead to a complete dissolution of the filament.

Generally, an electric field is applied to a CBRAM cell is with the help of a DC voltage applied across it as shown in Fig. 2. Set $(\mathrm{ON})$ and reset (OFF) states thus formed are nonvolatile, meaning they do not require any power supply to maintain an impedance state, and a power supply is required only to change an impedance state whenever desired.

A simple electrical equivalent model of such a CBRAM switch is very similar to an RC parallel network as shown in Fig. 2. (d), whose resistance $R_{M I M}$ is equal to measured filament resistance across the switch, and capacitance $C_{M I M}$ is equal to parallel plate capacitance calculated with respect to electrode cross section area, thickness of ion-conductor and its dielectric properties [11], [22], [27].
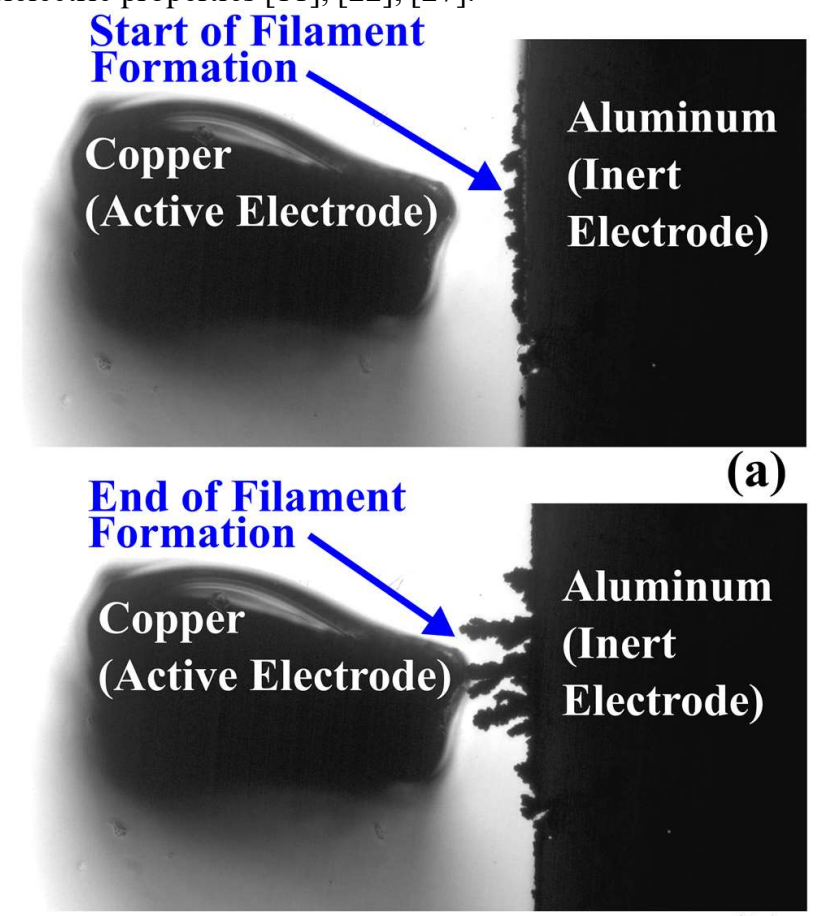

(b)

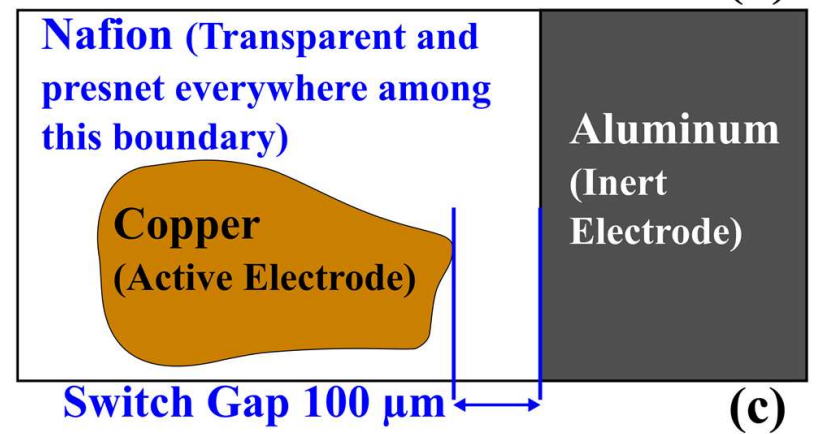

Fig. 3. Observation of filament formation in a CBRAM cell with copper/Nafion/aluminum switch layers on glass substrate [29]. (View: back side view on a glass substrate captured through an optical transmission microscope.) (a) Start of filament formation. (b) End of filament formation. (c) Sketch of CBRAM cell geometry, used in this experiment.

Polymer ion conductors like Nafion facilitates realization of CBRAM switches, and electronically reconfigurable RF devices with integrated CBRAM switches in an ambient lab environment outside 'clean room' conditions [11], [16], [27], [30]. Nafion is a flexible ionomer or ion conductor, whose layers could be easily formed by spin coating Nafion resin solution on most substrates. These layers could be air dried in 
ambient environment without the requirements of any high temperature or vacuum based processes as for the inorganic ion conductors discussed above. The optimized process for realization of Nafion based CBRAM RF switches are reported in [27] and [16] for rigid (like FR-4 Epoxy) as well as flexible substrates (like paper) respectively.

A group of CBRAM cells, fabricated on a classic copper cladded FR-4 epoxy substrate, finally with copper/Nafion/aluminum layers are depicted in Fig. 4.

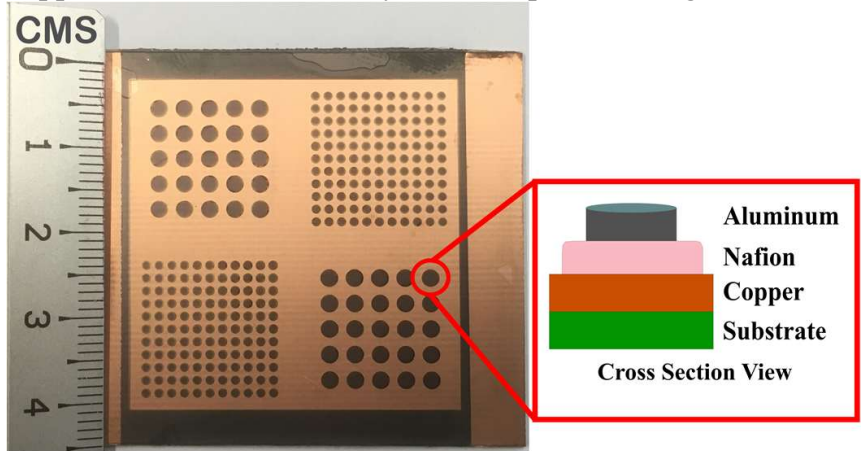

Fig. 4. Photograph of copper/Nafion/aluminum based CBRAM cells fabricated on FR-4 epoxy substrate, with inset showing the cross section view of material layers present. Please note that the Nafion layer is not visible on the photograph due to its transparency.

These cells are fabricated using a simple and optimized process reported in [26], explained as follows. Initially, the copper surface $(\sim 35 \mu \mathrm{m}$ thick) is cleaned using acetone to remove any impurities, and then Nafion resin solution [31] is spin coated everywhere at a preset rate for a given thickness (explained in following section II.A in detail). Next, button shaped aluminum layer depositions with thickness of the order of $500 \mathrm{~nm}$ to $1 \mu \mathrm{m}$ (as shown in Fig. 4) is done through thermal vapor deposition technique, using a dedicated nickel mask, with engraved apertures having dimensions of aluminum buttons, to complete the process. Here copper and aluminum are respectively the active and inert electrodes.

\section{A. Experiment on Optimization of Nafion Layer Thickness for CBRAM RF Applications}

In this section, we present the details of optimization of material properties of copper/Nafion/aluminum CBRAM switch for RF applications. In this study it is important to optimize material properties of a CBRAM switching cell to operate at considerably low resistances $(\sim<10 \Omega)$ in set $(\mathrm{ON})$ state and considerably high resistances (greater than few $\mathrm{k} \Omega$ ) in reset (OFF) states for good RF transmission and isolation performances. However, for memory applications (which is the parent field of CBRAM technology) only a considerable distinction between the states are acceptable $(\mathrm{k} \Omega$ and $\mathrm{M} \Omega)$ in most cases [32]. One of the critical factors that determine these resistance states is the ion conductor layer. As explained above, in this study, layers of ion conductor (Nafion) are formed through spin coating, and it is necessary to optimize a layer thickness of Nafion for obtaining a good level of set $(\mathrm{ON})$ and reset (OFF) state impedances. In this aspect, here we present some brief and relevant results of a basic experiment, to optimize the thickness of Nafion layer. Fig. 5 presents a few switching cycles of CBRAM cells as in Fig. 4, for different thickness of Nafion layers. DC pulses as reported in [16], [27] are utilized for operating the CBRAM switches in this experiment.

The chosen layer thicknesses of Nafion for this experiment are respectively $1400 \mathrm{~nm}$ and $600 \mathrm{~nm}$. As indicated above, a commercial Nafion resin solution [31], is spin coated at a rate of $500 \mathrm{RPM}$ for $30 \mathrm{~s}$ and then dried on a hot plate at $45^{\circ} \mathrm{C}$ for $60 \mathrm{~s}$ to achieve a layer thickness of $600 \mathrm{~nm}$. This process is repeated, one on top of each layer for three times to achieve a layer thickness of $1400 \mathrm{~nm}$. Layer thicknesses are then confirmed to be as expected using a Dektak 150 mechanical profilometer to ensure quality of samples. This process is optimized through several trial and errors and is reported in [27].

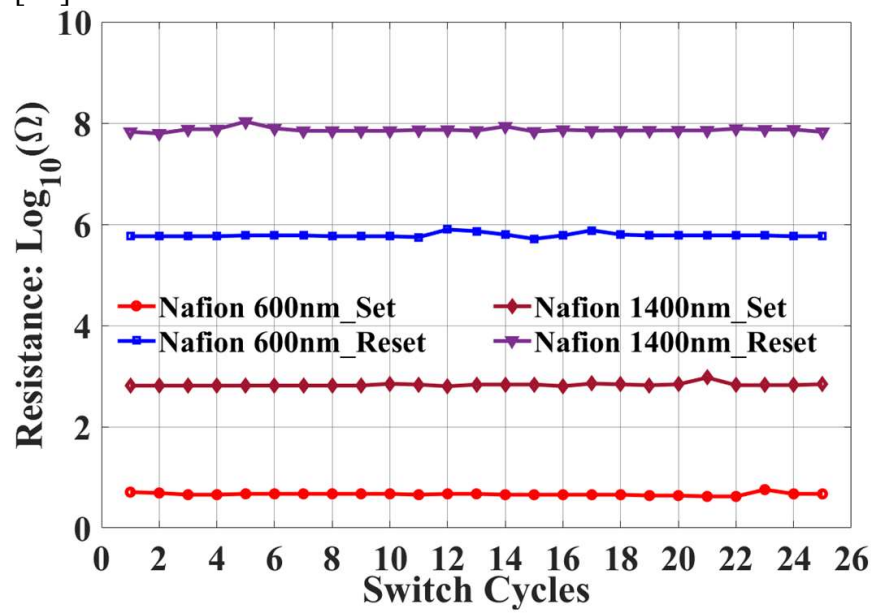

Fig. 5. Typical 25 switching cycles of copper/Nafion/aluminum CBRAM switches similar to as shown in Fig. 4 for different layer thickness of ionconductor (Nafion).

From Fig. 5, one could see the importance of optimization of ion-conductor layer thickness. CBRAM cells with $1400 \mathrm{~nm}$ layer of Nafion are seen to exhibit impedance of the order of $>$ $500 \Omega$ and $60 \mathrm{M} \Omega$ respectively for set $(\mathrm{ON})$ and reset $(\mathrm{OFF})$ states, which are not usable (due to higher ON state resistances) for a classic RF switching application.

However in contrast to this, CBRAM cells with $600 \mathrm{~nm}$ layer of Nafion are seen to exhibit impedances of the order of $\sim 4 \Omega$ and $1 \mathrm{M} \Omega$ respectively for set $(\mathrm{ON})$ and reset (OFF) sates. These impedance values are more considerable for RF switching applications, and hence, a layer thickness of $600 \mathrm{~nm}$ for ion-conductor layer is adopted throughout in this study. It is obvious that further lower layer thicknesses would provide better low impedances in set (ON) states, but are however not attempted in this optimization, to limit the possible high OFF (reset) state capacitances, and quick dielectric breakdown of the switches.

CBRAM switches fabricated using similar techniques in our group are seen to be functional even after 2300 cycles of operations [26]. This is considered to be a good value for such crude fabrication techniques, in this infancy state, and notably without utilizing any clean room facilities. CBRAM switches fabricated using this technique is also seen to exhibit a considerable stability of achieved impedance states of more than 1000 minutes as reported in [30]. 


\section{DESIGN OF ELECTRONICALLY REWRITABLE CHIPLESS RFID TAGS}

In this section, we present the design of an electronically rewritable resonator for chipless RFID applications, and the proposed realization technique for fabrication of a fully printed electronically rewritable chipless RFID tag based on three such resonator structures, on flexible PET laminates.

\section{A. Rewritable Resonator Design}

REP for the proposed electronically rewritable chipless RFID tag is inspired from a simple closed loop resonator [33], and is integrated with two CBRAM RF switches by adapting the geometry. Fig. 6 shows the topology of a single rewritable REP with two integrated CBRAM switches. Geometric dimensions of this resonator are given in Table 1.

CBRAM switches are integrated into this design by sandwiching a $600 \mathrm{~nm}$ layer of Nafion among the trace width marked by $W 1$ and $W 2$ as shown in cross section view in Fig. 6. Polyethylene terephthalate (PET) with a layer thickness of $50 \mu \mathrm{m}$ is chosen as the substrate for this design.
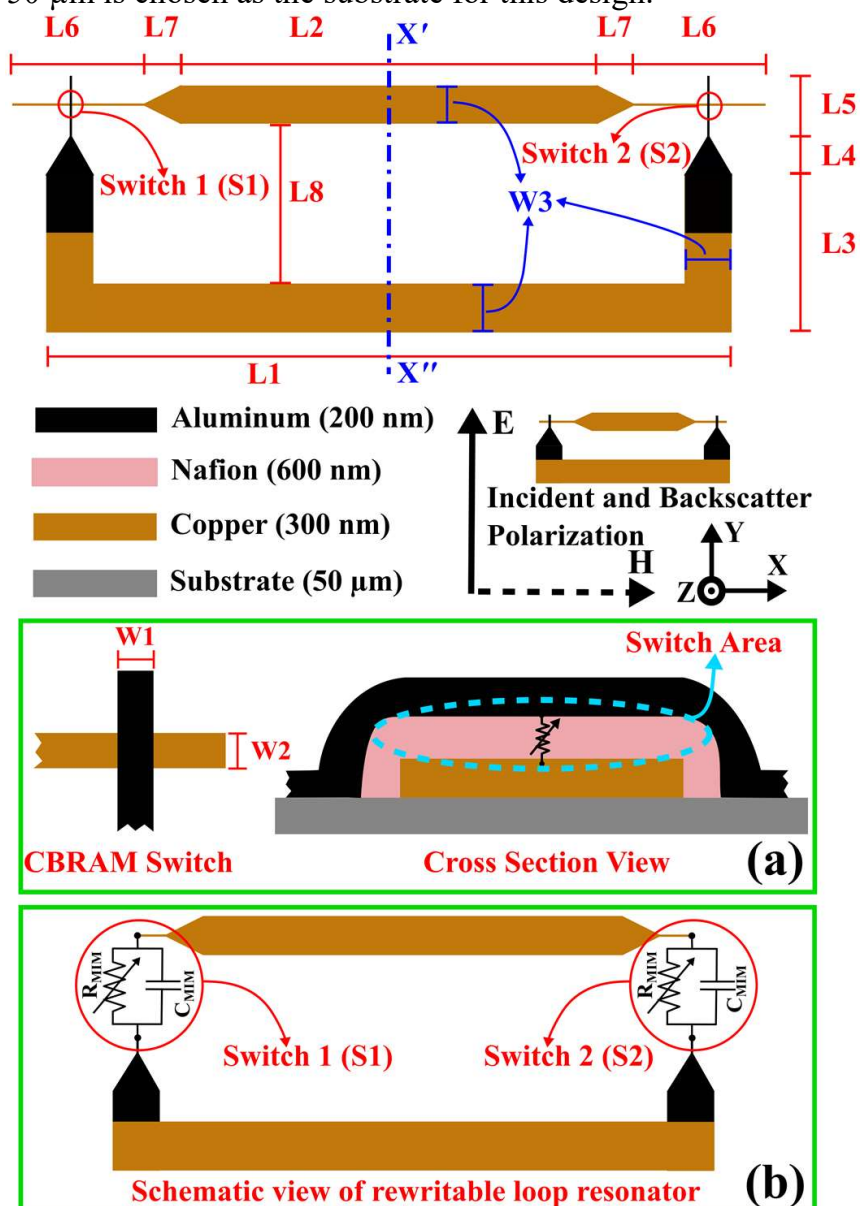

Fig. 6. Geometry of proposed electronically reconfigurable loop resonator with integrated CBRAM switches. Inset (a) in green border shows zoomed view of integrated CBRAM switch and its cross section view. Inset (b) in green border shows the schematic view of connection of integrated CBRAM switches to the loop resonator. (All layer thicknesses are indicated in legends. Please note that this image is only a representative illustration and is not up to scale of real model.)

In this section, full-wave electromagnetic simulation studies of this resonator are presented. CST microwave studio is utilized for modeling and simulation of the presented design. An electromagnetic plane wave with incident polarization as shown in Fig. 6 is used as excitation, and an RCS probe (an inbuilt functionality of CST microwave studio) placed in farfield of the model is used to obtain the backscatter response. CBRAM switches in the design are modeled as their respective electrical models (RC parallel networks as shown in Fig. 2) and their values are chosen using knowledge from previous realizations [11], [27], [30], and are given in Table 1.

This is done to lower the simulation time, by avoiding very fine mesh cell size for modeling naturally thin switch layers. Metallic layers in the design are modeled using 'ohmic sheet' option available with CST Microwave Studio. Sheet resistances obtained in experiments are used with this model $(0.379 \Omega / \mathrm{sq}$, and $0.505 \Omega / \mathrm{sq}$ respectively for copper and aluminum). Such thin metallic layers of the order of $200 \mathrm{~nm}$ and $300 \mathrm{~nm}$ for aluminum and copper respectively, are used in the design, is due to the characteristics of chosen fabrication process (thermal transfer printing) explained in following section III.C.

TABLE 1

DIMENSIONS OF ELECTRONICALLY REWRITABLE LOOP RESONATOR IN FIG. 6.

\begin{tabular}{|c|c|c|c|}
\hline Parameter & Value & Parameter & Value \\
\hline $\mathrm{L} 1$ & $74 \mathrm{~mm}$ & $\mathrm{~L} 7$ & $2.9 \mathrm{~mm}$ \\
\hline $\mathrm{L} 2$ & $60 \mathrm{~mm}$ & $\mathrm{~L} 8$ & $3 \mathrm{~mm}$ \\
\hline $\mathrm{L} 3$ & $4.5 \mathrm{~mm}$ & $\mathrm{~W} 1$ & $0.2 \mathrm{~mm}$ \\
\hline $\mathrm{L} 4$ & $1 \mathrm{~mm}$ & $\mathrm{~W} 2$ & $0.2 \mathrm{~mm}$ \\
\hline $\mathrm{L} 5$ & $3 \mathrm{~mm}$ & $\mathrm{~W} 3$ & $3 \mathrm{~mm}$ \\
\hline $\mathrm{L} 6$ & $4.15 \mathrm{~mm}$ & $\mathrm{R}_{\mathrm{MIM}}(\mathrm{ON} / \mathrm{Set})$ & $1 \Omega$ to $10 \Omega$ \\
\hline $\mathrm{R}_{\mathrm{MIM}}$ (OFF/Reset) & $100 \mathrm{k} \Omega$ to $1 \mathrm{M} \Omega$ & $\mathrm{C}_{\mathrm{MIM}}$ & $1.5 \mathrm{pF}$ to $1.8 \mathrm{pF}$ \\
\hline
\end{tabular}

Presented loop resonator is capable of resonating at three different frequencies and encodes three states or codes as shown in Fig. 7 depending on ON/OFF (set/reset) state of the integrated switches. These states are, code 0 with resonance at $2.04 \mathrm{GHz}$, when both switches $\mathrm{S} 1$ and S2 are in high impedance, or OFF (reset) states. Code 1, whose resonance is at $1.94 \mathrm{GHz}$, when $\mathrm{S} 1$ is $\mathrm{ON}$ (set) and $\mathrm{S} 2$ is OFF (reset), or vice versa, due to symmetry of loop along the center $\left(X^{\prime}-X^{\prime \prime}\right)$ as shown in Fig. 6. And code 2, with resonance at $1.82 \mathrm{GHz}$, when both $\mathrm{S} 1$ and $\mathrm{S} 2$ are in $\mathrm{ON}$ (set) state.

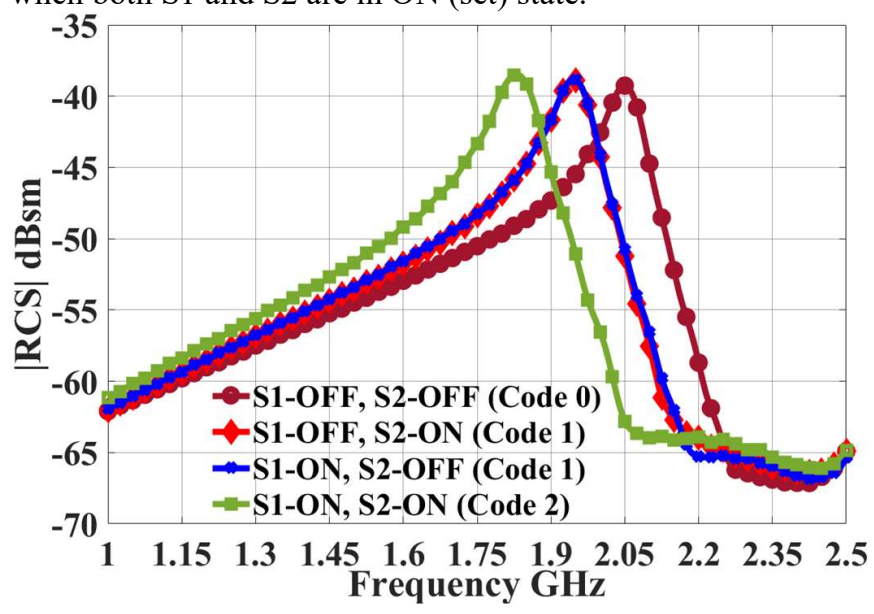

Fig. 7. Simulated (full wave) RCS backscatter magnitude of electronically rewritable loop resonator in Fig. 6 with dimensions in Table 1 for different switch configurations of integrated CBRAM switch.

Proposed REP exhibits a surface current pattern across the perimeter of the loop, similar to a full wavelength resonance, 
for each switch combination, in simulation studies (full wave) at their respective resonance frequencies, as shown in Fig. 8, for all possible switch combinations. The surface current pattern at all resonance frequencies are similar since the integrated CBRAM switch tunes the electrical length of the corresponding loop forcing it to resonate at the corresponding frequencies indicated above. The working mechanism of this electronically rewritable loop resonator is explained in detail in section III.B. Electrical Equivalent Modeling.

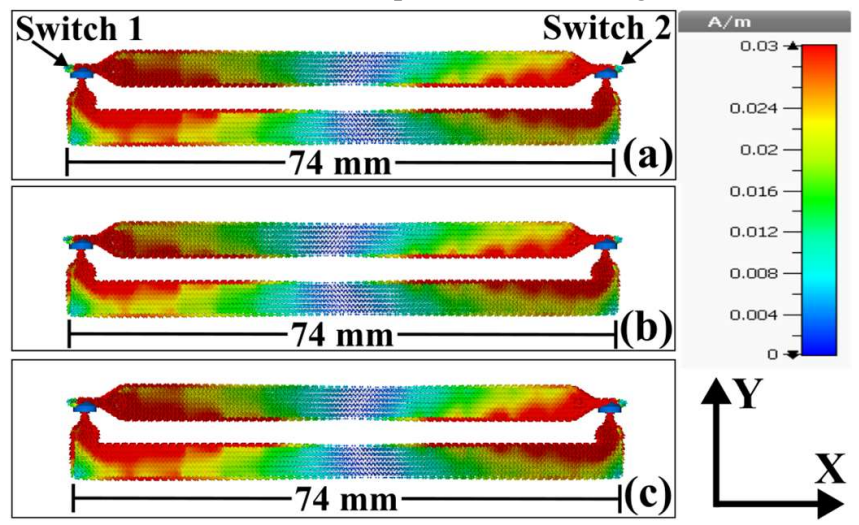

Fig. 8. Surface current patterns of proposed electronically rewritable loop resonator at their respective resonance frequencies for all possible switch combinations. (a) State $0-2.04 \mathrm{GHz}$. (b) State $1-1.94 \mathrm{GHz}$. (c) State $2-$ $1.82 \mathrm{GHz}$.

Observed characteristics of the proposed resonator explained above are a considerable enhancement in comparison to our previous studies [11], [16]. In which one resonator could represent only one bit or two states, whereas in this case it is more than one bit with three states per REP.

Moreover, the chosen resonator design (loop resonator) is apparently acknowledged to provide a higher RCS level than a ' $\mathrm{C}$ ' like structure (used in our previous design [11], [16]) operating at the same resonance frequencies [1], [33]. This is a remarkable and greatly desired improvement, which makes the proposed design more compatible with low metallic layer thicknesses and higher sheet resistivity, which is however a limiting characteristic, at least for the mean time, with the chosen fabrication technique for this realization, which is explained in section III.C.

Next, we present the geometry of an electronically rewritable chipless RFID tag, that consist of three loop resonators as discussed above, having different lengths to resonate at different frequencies, and is shown in Fig. 9. Dimensions of this design are summarized on Table 2.

$$
\text { TABLE } 2
$$

DIMENSIONS OF ELECTRONICALLY REWRITABLE CHIPLESS RFID TAG SHOWN IN FIG. 9 (PHOTOGRAPH OF FABRICATED TAG IS GIVEN IN FIG. 13).

\begin{tabular}{|c|c|c|c|}
\hline Parameters & Loop 1 & Loop 2 & Loop 3 \\
\hline L1 & $74 \mathrm{~mm}$ & $52.76 \mathrm{~mm}$ & $44 \mathrm{~mm}$ \\
\hline L2 & $60 \mathrm{~mm}$ & $38.77 \mathrm{~mm}$ & $30 \mathrm{~mm}$ \\
\hline Parameter & Value & Parameter & Value \\
\hline D & $10 \mathrm{~mm}$ & Ls & $100 \mathrm{~mm}$ \\
\hline Ws & $85 \mathrm{~mm}$ & \multicolumn{3}{|l}{} \\
\hline All other dimensions are same as given in Fig. 6 and Table 1.
\end{tabular}

This particular tag with three rewritable loops could represent a total of 27 coded states depending on chosen switch combination (ON/OFF) of each resonator. Simulated (full wave) RCS backscatter response of this model is depicted in Fig. 10, for some selected states of switch combinations of integrated CBRAM switches. All 27 states follow these typical bandwidth and RCS magnitude characteristics, and are however omitted from this plot to keep it concise.

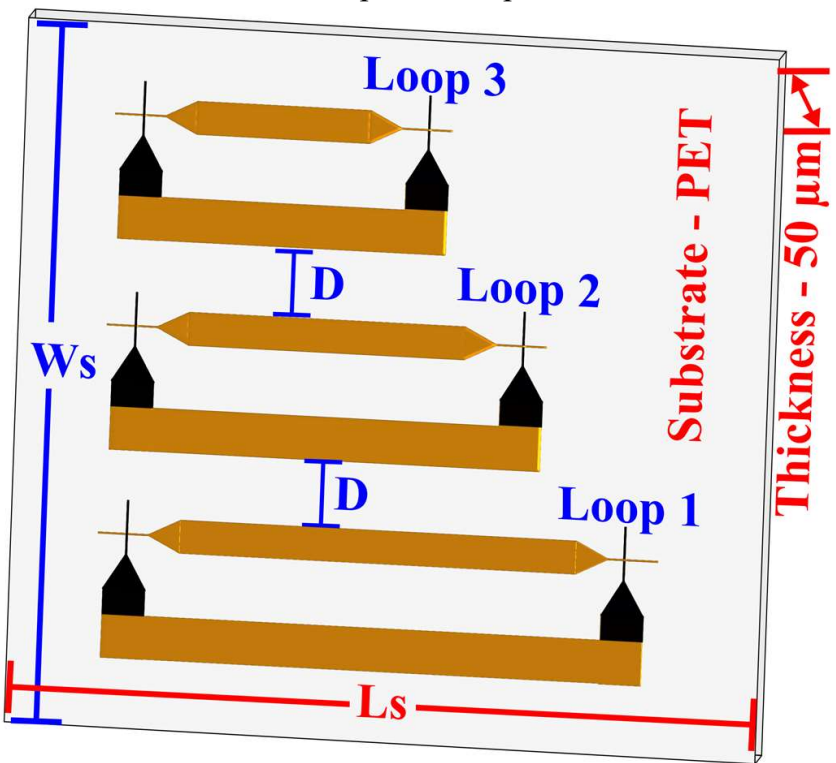

Fig. 9. Geometry of proposed electronically rewritable chipless RFID tag with integrated CBRAM switches.

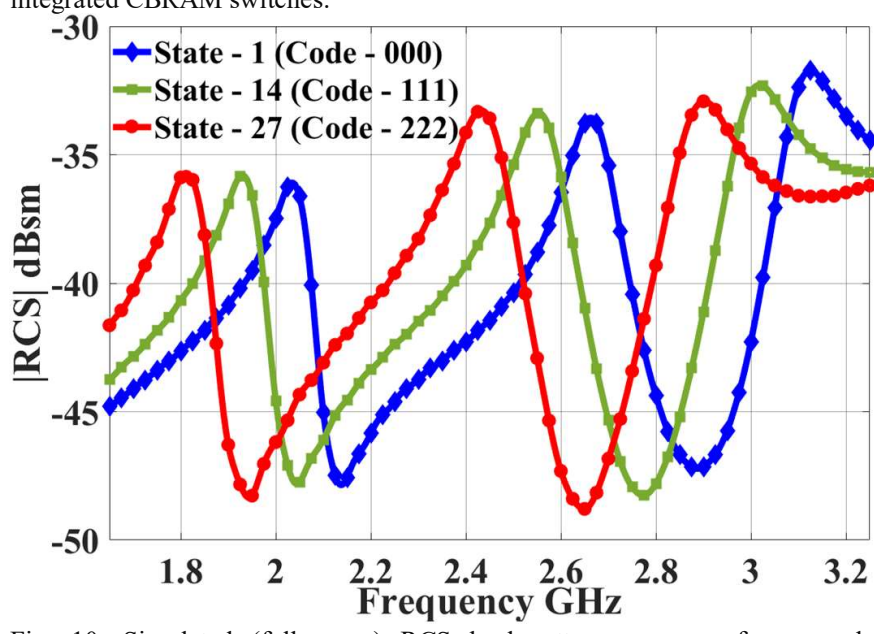

Fig. 10. Simulated (full wave) RCS backscatter response of proposed electronically rewritable chipless RFID tag in Fig. 9, for some selected switch combinations of integrated CBRAM switches. The code state represents switch states of REPs in the order of Loop 1,2 and 3 respectively.

\section{B. Electrical Equivalent Modeling}

In this section a simple electrical equivalent model of proposed electronically rewritable chipless RFID tag is presented. This model explains the functioning of integrated CBRAM switch in the presented electronically rewritable chipless RFID tag.

It is well known that the RCS backscatter response of a multi scatterer based chipless RFID tag, is an interference pattern, of two principle modes of scattering called the structural mode and antenna mode [34] in time domain. Structural mode response is a wide band resonant response as an effect of immediate backscatter of incident signal from reflective parts of the tag similar to an optical reflection, and antenna mode is a sum of time cleaved and narrow band 
resonant response of each resonator present on the tag. With respect to this analogy an electrical model of a multi scatterer based chipless RFID tag could be approximated with a group of RLC serial resonant circuits operating in parallel and excited with appropriated signal magnitudes and phase, respectively for the structural mode of whole of the tag, and antenna mode of each individual resonator present on the tag [1], [35].

In the presented case of rewritable chipless RFID tag with loop resonators with integrated CBRAM switches in Fig. 9, the electrical model could be approximated as shown in Fig. 11. Here the electrical model of each CBRAM switch (which is an RC parallel network as explained in section II with reference to Fig. 2), which is in series with the perimeter of loop resonators in the physical model (as shown in Fig. 6), is added in series with the antenna mode RLC network, for each corresponding loop resonator present on the tag. Resistance values of CBRAM cell's electrical model are chosen from the experimentally obtained filament resistance values for corresponding $\mathrm{ON}$ and $\mathrm{OFF}$ states. The capacitance values in this electrical model are evaluated from the physical model of CBRAM cells, based on calculated parallel plate capacitance values, from the cross section area of electrodes, thickness of Nafion (ion-conductor) and its dielectric properties [36]. Other component values are chosen based on simple equations of electrical engineering defining RLC series resonances, based on resonance frequency values obtained in full-wave electromagnetic simulation, and are then optimized accordingly to match the simulation results. This model is thus refined using PSpice circuit simulator to best fit the full-wave electromagnetic simulation response.

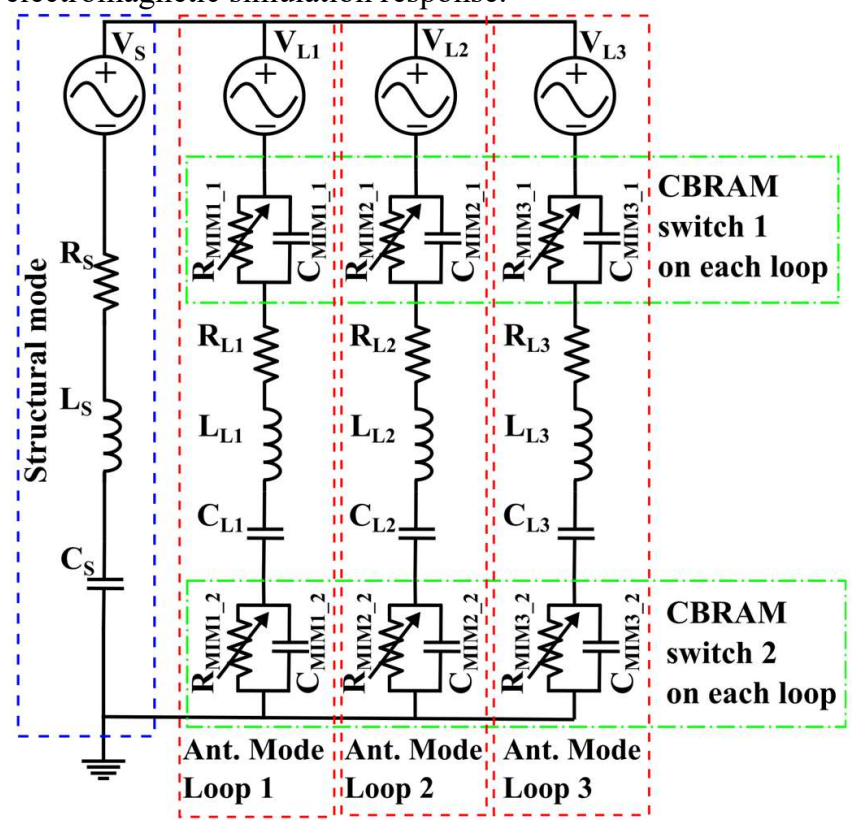

Fig. 11. Electrical equivalent model of proposed electronically rewritable chipless RFID tag.

Component parameter values of this electrical model are depicted in Table 3. Results of this electrical model for two of the states (these two states are randomly picked and other results are omitted to maintain brevity) are depicted in Fig. 12 along with simulation (full wave) results for comparison. One could observe in Fig. 12 that results of electrical model are in good agreement with the full wave simulation results (the results for other combinations which are not presented herewith, also maintains a similar tight agreement), and this validates the accuracy of proposed model.

TABLE 3

COMPONENT PARAMETER VALUES OF ELECTRICAL EQUIVALENT MODEL DEPICTED IN FIG. 11

\begin{tabular}{|c|c|c|c|}
\hline Parameter & Value & Parameters & Value \\
\hline $\mathrm{R}_{\mathrm{s}}$ & $15 \Omega$ & $\mathrm{R}_{\mathrm{MIM} 21}$ & $1 \Omega / 1 \mathrm{M} \Omega$ * \\
\hline $\mathrm{R}_{\mathrm{L} 1}$ & $16 \Omega$ & $\mathrm{R}_{\text {MIM2 2 }}$ & $1 \Omega / 1 \mathrm{M} \Omega *$ \\
\hline $\mathrm{R}_{\mathrm{L} 2}$ & $18 \Omega$ & $\mathrm{R}_{\text {MIM3_1 }}$ & $1 \Omega / 1 \mathrm{M} \Omega$ * \\
\hline $\mathrm{R}_{\mathrm{L} 3}$ & $20 \Omega$ & $\mathrm{R}_{\mathrm{MIM} 32}$ & $1 \Omega / 1 \mathrm{M} \Omega$ * \\
\hline $\mathrm{L}_{\mathrm{S}}$ & $0.03 \mathrm{nH}$ & $\mathrm{C}_{\text {MIM1 1 }}$ & $1.6 \mathrm{pF}$ \\
\hline $\mathrm{L}_{\mathrm{L} 1}$ & $38 \mathrm{nH}$ & $\mathrm{C}_{\text {MIM1 } 2}$ & $1.6 \mathrm{pF}$ \\
\hline $\mathrm{L}_{\mathrm{L} 2}$ & $28.2 \mathrm{nH}$ & $\mathrm{C}_{\text {MIM2_1 }}$ & $1.55 \mathrm{pF}$ \\
\hline $\mathrm{L}_{\mathrm{L} 3}$ & $25 \mathrm{nH}$ & $\mathrm{C}_{\mathrm{MIM} 2 \_2}$ & $1.55 \mathrm{pF}$ \\
\hline $\mathrm{C}_{\mathrm{s}}$ & $0.01 \mathrm{pF}$ & $\mathrm{C}_{\mathrm{MIM} 31}$ & $1.55 \mathrm{pF}$ \\
\hline $\mathrm{C}_{\mathrm{L} 1}$ & $0.2 \mathrm{pF}$ & $\mathrm{C}_{\mathrm{MIM} 32}$ & $1.55 \mathrm{pF}$ \\
\hline $\mathrm{C}_{\mathrm{L} 2}$ & $0.15 \mathrm{pF}$ & $\mathrm{V}_{\mathrm{S}}$ & $100 \mathrm{mV} / 0^{\circ}$ \\
\hline $\mathrm{C}_{\mathrm{L} 3}$ & $0.12 \mathrm{pF}$ & $\mathrm{V}_{\mathrm{L} 1}$ & $1.5 \mathrm{mV} / 0^{\circ}$ \\
\hline $\mathrm{R}_{\text {MIM1 1 }}$ & $1 \Omega / 1 \mathrm{M} \Omega *$ & $\mathrm{~V}_{\mathrm{L} 2}$ & $2.5 \mathrm{mV} / 0^{\circ}$ \\
\hline $\mathrm{R}_{\text {MIM1 } 2}$ & $1 \Omega / 1 \mathrm{M} \Omega *$ & $\mathrm{~V}_{\mathrm{L} 3}$ & $3 \mathrm{mV} / 0^{\circ}$ \\
\hline
\end{tabular}

Working mechanism of the presented electronically rewritable chipless RFID tag with CBRAM switches could be explained as follows with reference to the proposed electrical model in Fig. 11. For this (for simplicity) we consider only one of the loop resonators present on the tag. Say for instance, in loop 1, initially, that both the CBRAM switches are in ON (set) state hence $R_{M I M 1 \_1}$ and $R_{M I M 1 \_2}$ are at low resistance state $(\sim 1 \Omega)$.

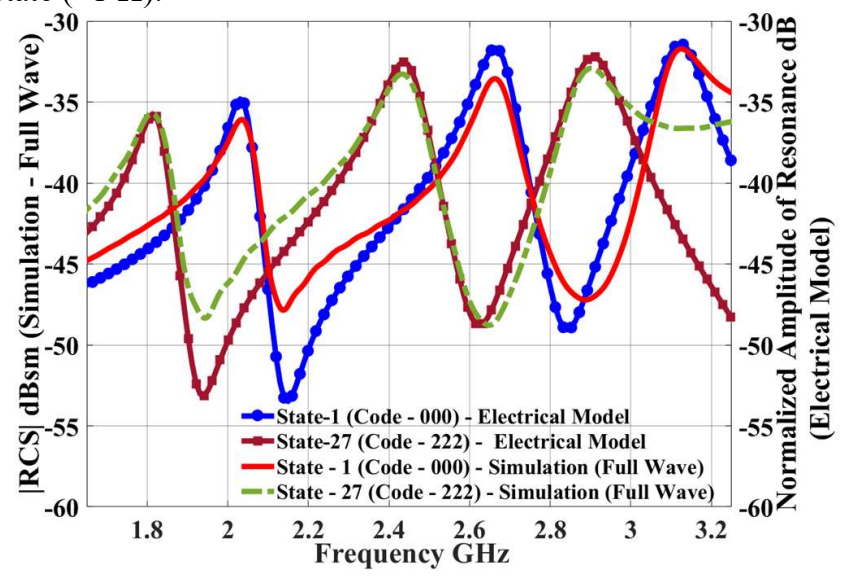

Fig. 12. Response of simulated electrical model in Fig. 11 for presented electronically rewritable chipless RFID tag along with simulated (full wave) RCS backscatter response for comparison.

At this time the capacitance $C_{M I M 1 \_1}$ and $C_{M I M 1 \_2}$ has no effect as it is bypassed by the corresponding resistance $R_{M I M 1_{-} 1}$ or $R_{M I M 1_{-} 2}$, which is similar to a short circuit, and the resonance depends only on $R_{L 1}, L_{L 1}$, and $C_{L 1}$ to represent code 2. However, if a CBRAM switch (or both) is in OFF (reset) state i.e. if $R_{M I M 1 \_1}$ or $R_{M I M 1_{-} 2}$ or both are in high resistance state $(\sim 1 \mathrm{M} \Omega)$, this is similar to an open circuit and 
adds the respective capacitance $C_{M I M 1 \_1}$, or $C_{M I M 1 \_2}$, or both, correspondingly in series to $C_{L 1}$ thus reducing the net capacitance of the resonant circuit, and forces it to resonate at a higher frequency, to represent code 1 or 0 respectively. The state symmetry when one of the switches is ON and other is OFF and vice versa, as explained in section III.A applies also to this circuit.

One should note that the presented electrical model is a combined system that takes into consideration the instantaneous state of integrated CBRAM switches on each REPs based on its filament resistance, and the resonance response of individual geometries, as well as the mutual coupling among the resonators for the final tag design with which the electrical model is optimized to a best fit. Hence, it would be simple and straight forward to compensate mutual coupling effect on a tag due to any re-arrangement of resonators (REPs) with a small increment/decrement factor on corresponding component values of each RLC networks present on the electrical model, for each addition/deletion/rearrangement/geometrical-modification of resonator elements on the electronically rewritable chipless RFID tag, for a best fit of this model with experimentally observed characteristics of the tag.

Moreover an interesting accomplishment with the presented approach is that there are almost no requirements for rearrangement of position of REPs or modification of resonator geometries for encoding a desired code from a given set of possible combinations in contrast to classic mechanical reconfiguration approaches (similar to as explained in the Introduction section). Instead, one just needs to change the impedance state of appropriate CBRAM switches on the respective resonators, using appropriate DC pulses. Thus there would be practically very less or no detuning related to mutual coupling with the presented types of electronically rewritable chipless RFID tags.

It is true that the presented model could only represent principal harmonic mode of resonators present on the proposed tag. Nevertheless, this is a very simple and effective way to predict the behavior of a tag for a chosen combination of CBRAM switches, and also to explain the working mechanism of presented electronically rewritable chipless RFID tag based on integrated CBRAM switches. In addition, as indicated above in the introduction section, this model could save an appreciable amount of time required with lengthy full wave electromagnetic simulations to determine behavior of all code states from a required number of combinations desired for a tag application. In our approach, one single design and two unique parameters (switch resistance states - $R_{M I M}$ of each of two switches) are used to control the electromagnetic signature (three resonance state per resonator) of a given REP. Hence based on a simple PSpice or electrical model (devised only one time for a genre of proposed tags), one could easily compute all different possible combinations just by changing the resistance values of corresponding switches on each resonator. It means that a similar model like in Fig. 11 and Table 3 has to be computed only once and then all possible different combinations could be computed by just changing the required parameters out of $N$ resistance values $\left(N=2 R_{M I M}\right.$ per REP times number of REP) present with the model. A simple numerical program could be devised using this concept which could accept a required tag ID (or switch combinations) and generate the corresponding RF signature (RCS response), using the PSpice model. Thus with this approach, it is possible to accurately compute RCS response for all or any of desired possible combinations, of the tag using an optimized electrical model.

Only minimum full wave electromagnetic simulations to define basic electromagnetic behavior of each resonator present on the tag are required in such a case, so as to accurately define the electrical model. This would be similar to as given in Fig. 10 which shows all possible resonant states of each resonator present on the proposed tag, however limited to three (which is just the number of code states per resonator used in this application) full wave electromagnetic simulation runs.

A detailed study on boundary conditions and resonant modes of loop resonators from which the presented resonators are evolved, and their analysis based on RCS backscatter response, through transmission line approach is reported in [37]. In which loop resonators similar to as presented herewith are considered as coplanar stripline (CPS) segments with short circuit across both their ends. This is a simple analogy to understand the resonant modes and boundary conditions of presented loops as well. Such an analysis maybe adopted for highly accurate modeling of presented electronically rewritable chipless RFID tags with loop resonators, for studies related to higher order modes and coupling effects.

\section{Fabrication Technique}

In this section, we present the fabrication techniques for the electronically rewritable chipless RFID tag discussed above. Fig. 13 shows the photograph of realized tag with three resonators.

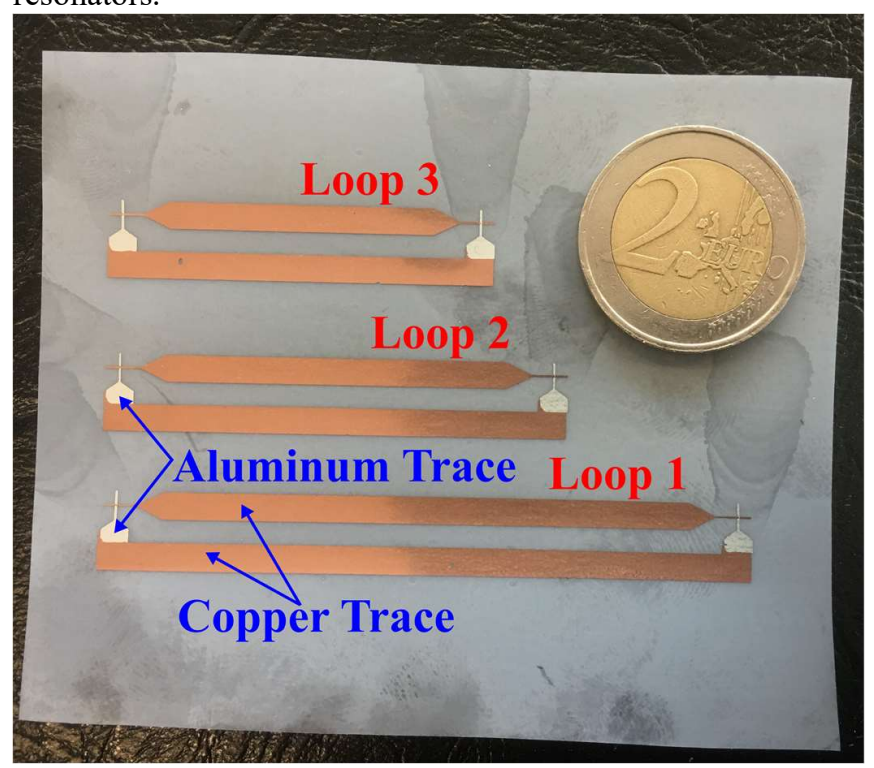

Fig. 13. Photograph of fully printed electronically rewritable chipless RFID tag with integrated CBRAM switches, on flexible PET substrate.

Metallic parts of the realized tag are fully printed, including 
one Nafion coating step. ZT610 industrial thermal transfer printer [38] is utilized for this realization. Thermal transfer printing of metallic traces is an emerging process in which a group of heated pixel shaped pistons or print heads are used to selectively transfer a given pattern from a pre-inked (or metal coated) former to a substrate. Here we use a 4 inch wide sheet of $50 \mu \mathrm{m}$ thick ordinary PET sheet as the substrate and custom supplied copper and aluminum thermal transfer ribbons for the metal trace. Initially one of the electrodes (copper or aluminum) is printed like in an ordinary printer from an actual scale PDF file. Then the reference position for next layer print is marked on the PET reel, and this trace is spin coated with Nafion resin solution as in [27] to obtain a layer thickness of around $600 \mathrm{~nm}$, optimized for a good ON/OFF (set/reset) impedance. After this step, the PET sheet is loaded back to the printer at the predefined reference position and the opposite electrode (aluminum or copper correspondingly) is printed to complete the fabrication process. The printed tags are then inspected under an optical microscope for any possible defects and are then retained for experimental studies.

Metallic trace thicknesses on the printed tags were measured using Dektak-150 mechanical profilometer and were found to be around $200 \mathrm{~nm}$ for aluminum and $300 \mathrm{~nm}$ for copper. Sheet resistances of the printed copper and aluminum samples are measured to be respectively, $0.379 \Omega / \mathrm{sq}$, and $0.505 \Omega /$ sq.

As indicated above in section II, the integrated CBRAM switches in the proposed tag are operated using DC pulses as reported in [16], [27]. These pulses are applied to the switches by attaching ordinary ohmic contact probes (custom made using conducting wires) to adjacent metallic parts (appropriate electrodes). However, as the chosen structure of utilized REP is that of a closed loop, to avoid a potential short circuit, there would have been a requirement of DC blocking capacitors, either as a lumped element or as a distributed element on the traces to operate one switch, when the other is in a low impedance state $(\mathrm{ON})$ to avoid a potential short circuit of applied DC pulses. Fortunately, since the chosen structure is a closed loop that resonates at full wavelength mode, a minute cut of micrometer dimensions is made using a sharp scalpel, on one of the loop lengths $L 1$ or $L 2$ along the axis of symmetry as indicated in Fig. $6\left(X^{\prime}-X^{\prime}\right)$. This enables one to program the CBRAM switches individually without causing a short circuit at DC. Presence of a current null at this point as indicated in Fig. 8 prevents any disturbance to the expected RF performance, with this technique.

\section{RESUltS AND DisCUSSIONS}

\section{A. Experimental Observations}

Experiments to obtain RCS backscatter response of fabricated tags were done using Keysight N5222A Network Analyzer in bistatic setup using the calibration and data processing reported in [6]. Two open boundary quad ridged horn antennas $(0.8-12 \mathrm{GHz})$ operating in co-polarization to each other, and also to the tag (see Fig. 6 for polarization of tag), are used to interrogate and receive backscatter from the tag. This entire experimental setup is constituted in a semi anechoic environment whose photograph is given in Fig. 14.

As mentioned above this tag with three resonators, with two CBRAM switches each is capable of representing 27 distinct states, depending on the ON/OFF state of each switch. Table 4 summarizes the total number of possible coded states obtained with the realized rewritable chipless RFID tag, respectively for each switch combination state, in experiment. Experimentally obtained RCS backscatter response for some selected codes are given in Fig. 15. All possible combinations are obtained affirmatively in experiment as summarized on Table 4, but are omitted from this plot to maintain brevity.

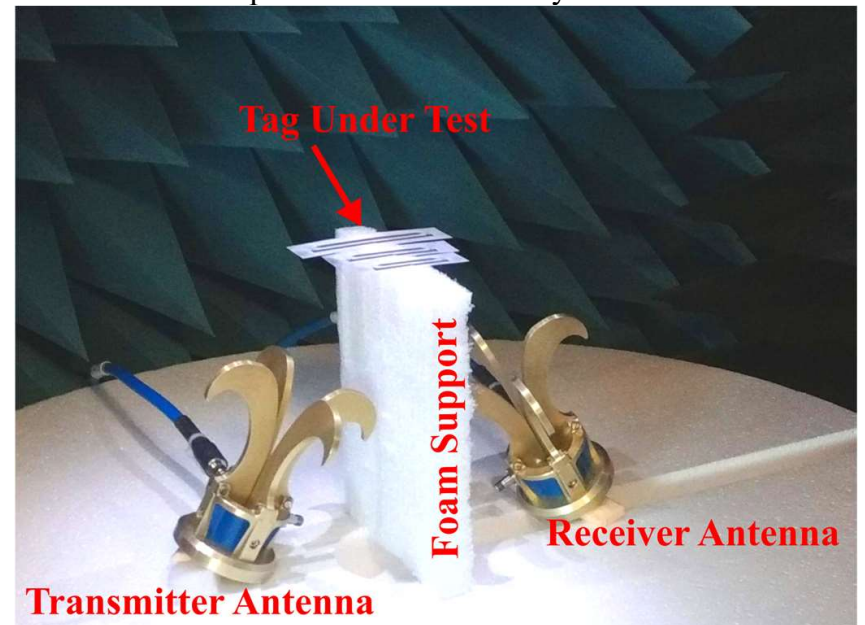

Fig. 14. Photograph of experimental setup used to capture the RCS backscatter response of presented electronically rewritable chipless RFID tag. (Network analyzer used is not visible in the photograph and is connected using appropriate RF transmission cables to the antennas.) TABLE 4

FREQUENCY OF RESONANCES OBSERVED IN EXPERIMENTALLY CAPTURED RCS BACKSCATTER RESPONSE OF ELECTRONICALLY REWRITABLE CHIPLESS RFID TAG IN FIG. 13 FOR ALL POSSIBLE SWITCH COMBINATIONS (CODES).

\begin{tabular}{|c|c|c|}
\hline $\begin{array}{c}\text { State } \\
\text { Number }\end{array}$ & $\begin{array}{c}\text { Code } \\
\text { (In order of Loop 1, } 2 \text { and 3) }\end{array}$ & $\begin{array}{c}\text { Resonance } \\
\text { Frequencies (GHz) } \\
\end{array}$ \\
\hline 1 & 000 & $2.002,2.668,3.154$ \\
\hline 2 & 001 & $1.988,2.670,3.038$ \\
\hline 3 & 002 & $2.002,2.670,2.926$ \\
\hline 4 & 010 & $1.988,2.562,3.152$ \\
\hline 5 & 011 & $1.988,2.562,3.044$ \\
\hline 6 & 012 & $1.988,2.562,2.926$ \\
\hline 7 & 020 & $1.988,2.472,3.150$ \\
\hline 8 & 021 & $1.988,2.472,3.034$ \\
\hline 9 & 022 & $1.986,2.470,, 2.926$ \\
\hline 10 & 100 & $1.908,2.672,3.150$ \\
\hline 11 & 101 & $1.911,2.670,3.038$ \\
\hline 12 & 102 & $1.911,2.670,2.926$ \\
\hline 13 & 110 & $1.918,2.562,3.150$ \\
\hline 14 & 111 & $1.906,2.562,3.040$ \\
\hline 15 & 112 & $1.911,2.562,2.926$ \\
\hline 16 & 120 & $1.911,2.465,3.149$ \\
\hline 17 & 121 & $1.914,2.470,3.038$ \\
\hline 18 & 122 & $1.910,2.466,2.926$ \\
\hline 19 & 200 & $1.820,2.672,3.152$ \\
\hline 20 & 201 & $1.820,2.670,3.038$ \\
\hline
\end{tabular}




\begin{tabular}{|l|l|l|}
\hline 21 & 202 & $1.822,2.672,2.930$ \\
\hline 22 & 210 & $1.820,2.562,3.149$ \\
\hline 23 & 211 & $1.820,2.562,3.038$ \\
\hline 24 & 212 & $1.820,2.562,2.926$ \\
\hline 25 & 220 & $1.818,2.450,3.138$ \\
\hline 26 & 221 & $1.826,2.472,3.036$ \\
\hline 27 & 222 & $1.814,2.452,2.926$ \\
\hline
\end{tabular}

Code combinations for each resonator based on switch 1 and 2 ON/OFF state (Ref. Fig. 6). Code $0-(\mathrm{OFF}$ OFF), Code $1-(\mathrm{ON}$ OFF, or OFF ON), Code 2 - (ON ON), for each loop (REP).

Overall size of presented electronically rewritable chipless RFID tag could be further reduced from the presented dimensions by employing resonators of smaller physical size, and resonating at a half or quarter wavelength current pattern in comparison to presented full wavelength loop resonators. On the other hand, the choice of loop resonators in the presented realization is to ensure adequate RCS magnitude level even with lower layer thicknesses and higher sheet resistances of printed metallic layers, achievable at present through the chosen thermal transfer printing process.

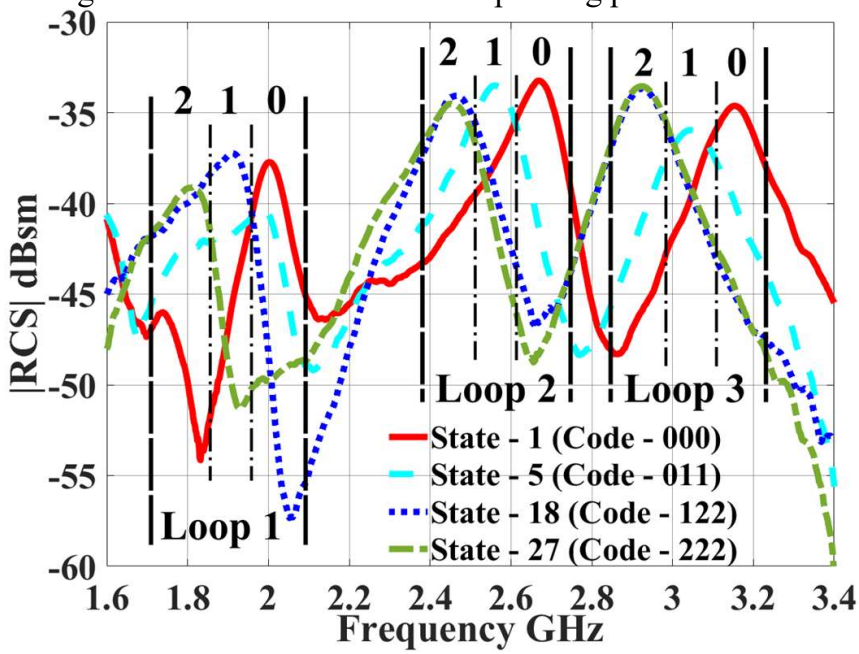

(a)

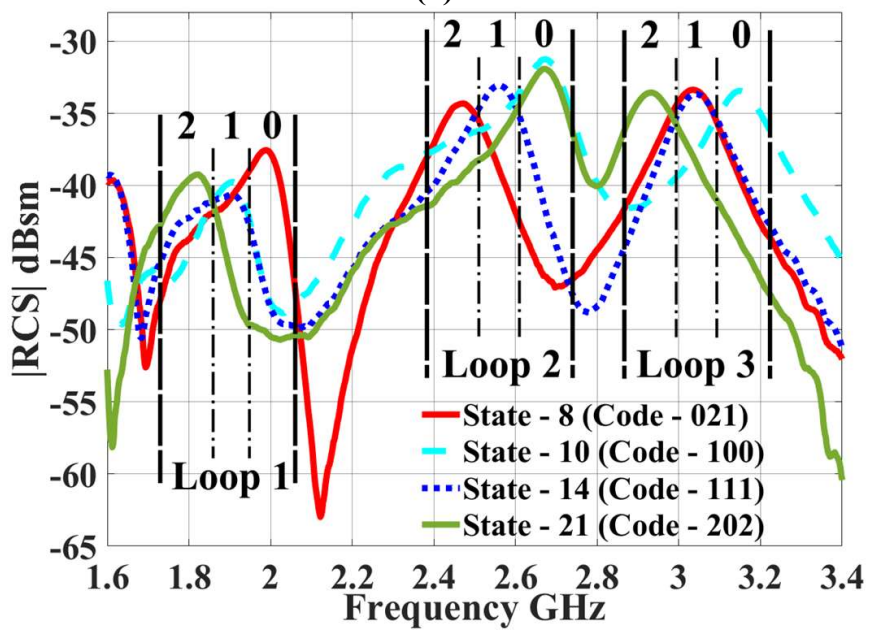

(b)

Fig. 15. Experimentally obtained RCS backscatter response of electronically rewritable chipless RFID tag presented in Fig. 13 for different switch combinations. (a) States 1,5,18,27. (b) States 8,10,14,21.

The currently available metallic thickness of 200 and 300 $\mathrm{nm}$ for aluminum and copper respectively, with the current fabrication/printing process (thermal transfer printing) used to realize the presented tag is well lower than the skin depth for copper $(1.43 \mu \mathrm{m})$ and aluminum $(1.83 \mu \mathrm{m})$ at the operating frequency $(1.7-3.2 \mathrm{GHz})$ of the tag. It is apparent from the experimentally obtained and simulated RCS magnitude levels of the presented rewritable chipless RFID tag, that a lower level of RCS magnitude values are obtained, in comparison to similar design with adequate thickness and conductivities for metal layers [6], [33]. This is due to the effect of below skin depth thicknesses, and observed higher sheet resistances (as indicated above in section III.C.), of printed metallic layers. An enhancement of 15 to $20 \mathrm{dBsm}$ in RCS peak magnitude levels are observed with the same electronically reconfigurable loop resonator geometries, but with adequate metallic thickness and sheet resistance similar to bulk metals (copper and aluminum correspondingly), in simulation studies. This plot is however omitted from herewith to keep the article concise.

Nevertheless, it is clear from Fig. 15 (a) and (b), that the presented tag exhibit distinct and readable RCS backscatter response for different switch combinations both in terms of operating frequency and magnitude, thus affirmatively proving feasibility of proposed idea, and chosen realization technique compatible with mass production through an industrial roll to roll process.

Detailed studies on techniques to improve the thickness and conductivity properties of printed metallic layers are in progress with the authors, with favorable outcomes, and are expected to deliver better performing electronically rewritable chipless RFID tags and RF/microwave devices, and are expected to be published in open literature in near future.

Maximum deviation of resonance frequencies, and obtained quality factor based on $3 \mathrm{~dB}$ bandwidth for each loop resonator present on the tag for all codes (switch combinations) are given in Table 5 for immediate information to the readers on the performance of presented electronically rewritable chipless RFID tag. The maximum deviation presented herewith is the maximum range of swing of resonance frequencies observed for each REP in the entire experiment, based on data given in Table 4 .

TABLE 5

MAXIMUM DEVIATION IN RESONANCE FREQUENCY, AND QUALITY FACTORS OF EACH RESONATOR, PRESENT ON THE ELECTRONICALLY RECONFIGURABLE CHIPLESS RFID TAG, FOR ALL SWITCH STATES.

\begin{tabular}{|c|c|c|c|}
\hline \multicolumn{4}{|c|}{ Maximum Deviation in Resonance Frequency } \\
\hline & Code 0 & Code 1 & Code 2 \\
\hline Loop 1 & $16 \mathrm{MHz}( \pm 8 \mathrm{MHz})$ & $8 \mathrm{MHz}( \pm 4 \mathrm{MHz})$ & $12 \mathrm{MHz}( \pm 6 \mathrm{MHz})$ \\
\hline Loop 2 & $4 \mathrm{MHz}( \pm 2 \mathrm{MHz})$ & $0 \mathrm{MHz}$ & $22 \mathrm{MHz}( \pm 11 \mathrm{MHz})$ \\
\hline Loop 3 & $16 \mathrm{MHz}( \pm 8 \mathrm{MHz})$ & $8 \mathrm{MHz}( \pm 4 \mathrm{MHz})$ & $4 \mathrm{MHz}( \pm 2 \mathrm{MHz})$ \\
\hline \multicolumn{4}{|c|}{ ' \pm ' value in brackets indicates the variation from an avg. center frequency. } \\
\hline \multicolumn{4}{|c|}{ Bandwidth (3 dB) and Quality Factor: (Average Values) } \\
\hline & Code 0 & Code 1 & Code 2 \\
\hline Loop 1 & $\begin{array}{c}\mathrm{BW}: 90 \mathrm{MHz} \\
\mathrm{Q}: 22.22\end{array}$ & $\begin{array}{c}\mathrm{BW}: 158 \mathrm{MHz} \\
\mathrm{Q}: 12.12\end{array}$ & $\begin{array}{c}\mathrm{BW}: 168 \mathrm{MHz} \\
\mathrm{Q}: 10.77\end{array}$ \\
\hline Loop 2 & $\begin{array}{c}\mathrm{BW}: 126 \mathrm{MHz} \\
\mathrm{Q}: 21.19\end{array}$ & $\begin{array}{c}\mathrm{BW}: 136 \mathrm{MHz} \\
\mathrm{Q}: 18.83\end{array}$ & $\begin{array}{c}\mathrm{BW}: 146 \mathrm{MHz} \\
\mathrm{Q}: 16.89\end{array}$ \\
\hline Loop 3 & $\begin{array}{c}\mathrm{BW}: 150 \mathrm{MHz} \\
\mathrm{Q}: 21.04\end{array}$ & $\begin{array}{c}\mathrm{BW}: 176 \mathrm{MHz} \\
\mathrm{Q}: 17.30\end{array}$ & $\begin{array}{c}\text { BW : } 148 \mathrm{MHz} \\
\text { Q : } 19.72\end{array}$ \\
\hline
\end{tabular}


Next we present the response of electrical model given in Fig. 11, for the realized electronically rewritable chipless RFID tag. Fig. 16 depicts the response of optimized electrical model of proposed chipless RFID tag along with experimentally obtained RCS backscatter response for comparison.

Parameter values for the proposed model remain essentially the same as in Table 3, except some small differences in component parametric values, summarized on Table 6 . These tiny variations in component parameter values could be justified by the possible fabrication tolerances between simulation model and experimental realization. Agreement among results of electrical model and experimentally obtained response once again validates the accuracy of proposed electrical model.

TABLE 6

COMPONENT PARAMETER VALUES OF ELECTRICAL MODEL DEPICTED IN FIG. 11, OPTIMIZED FOR EXPERIMENTALLY REALIZED ELECTRONICALLY REWRITABLE CHIPLESS RFID TAG IN FIG. 13.

\begin{tabular}{|l|l|l|l|}
\hline \multicolumn{1}{|c|}{ Parameter } & \multicolumn{1}{|c|}{ Value } & \multicolumn{1}{c|}{ Parameter } & \multicolumn{1}{c|}{ Value } \\
\hline $\mathrm{R}_{\mathrm{L} 3}$ & $18 \Omega$ & $\mathrm{C}_{\mathrm{MIM} 12}$ & $1.8 \mathrm{pF}$ \\
\hline $\mathrm{L}_{\mathrm{L} 2}$ & $27.6 \mathrm{nH}$ & $\mathrm{C}_{\mathrm{MIM} 21}$ & $1.8 \mathrm{pF}$ \\
\hline $\mathrm{L}_{\mathrm{L} 3}$ & $24.7 \mathrm{nH}$ & $\mathrm{C}_{\mathrm{MIM} 22}$ & $1.8 \mathrm{pF}$ \\
\hline $\mathrm{C}_{\mathrm{MIM} 11}$ & $1.8 \mathrm{pF}$ & \\
\hline All other component parameters are same as in Table 3. \\
\hline
\end{tabular}

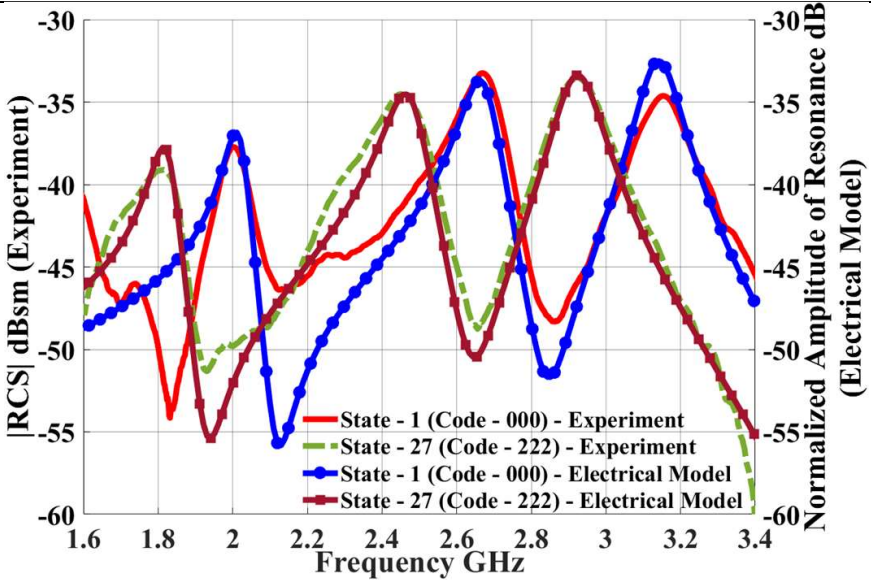

Fig. 16. Response of simulated electrical model in Fig. 11 for presented electronically rewritable chipless RFID tag in Fig. 13 along with experimentally obtained RCS backscatter response for comparison.

\section{B. Impedance Control of CBRAM Switches and their Effect} on RCS Backscatter Magnitude

One of an interesting question one could have now would be the effect of filament resistance of CBRAM switches, on the behavior of presented resonators. The following experiment is designed to give an insight to this aspect.

Here to keep the number of uncertainties minimum, we have used a loop resonator (REP) with only one CBRAM switch as shown in Fig. 17, and this resonator is printed as explained above.

In this experiment the integrated CBRAM switch is forced to different impedance states, by adjusting the magnitude of operating DC voltage pulses in a trial and error method to achieve different resistance states as indicated in Fig. 18. In this control experiment, in order to avoid the DC short-circuit issue as explained in section III.C, a part of the metallic loop is scratched using a sharp blade to make an open circuit and then connected using a conducting copper tape, after programing the CBRAM switch.

The obtained RCS backscatter responses which are an outcome of this experiment, are given in Fig. 18, and show a significant difference in corresponding RCS magnitude with respect to different impedance states. In other words the RCS resonance peaks are seen to be quantized to different magnitude levels, with different filament resistance values of integrated CBRAM switch. Such an interesting observation could be creatively utilized for achieving a better coding capacity of presented rewritable chipless RFID tags as follows.

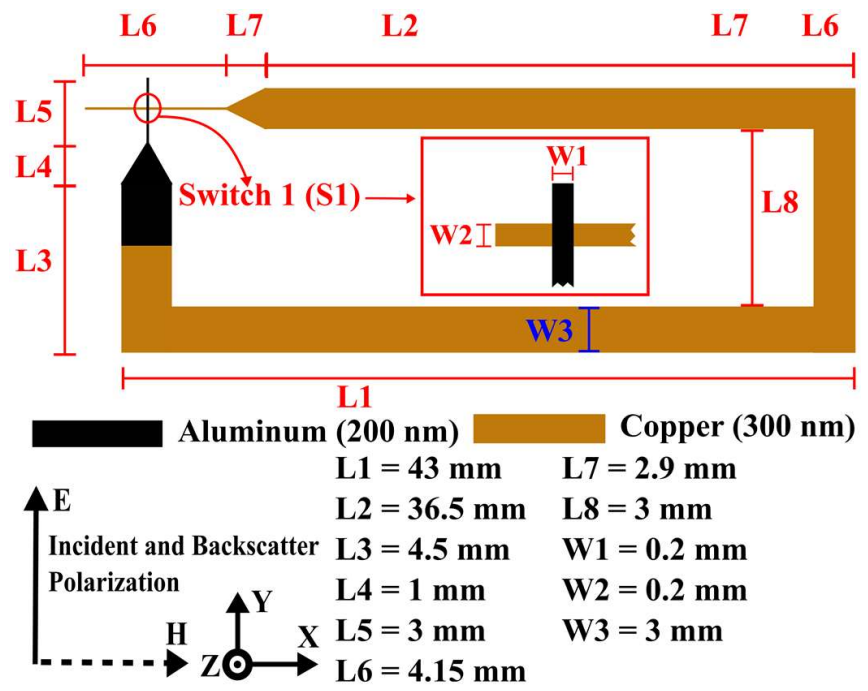

Fig. 17. Electronically reconfigurable loop resonator with one integrated CBRAM switch.

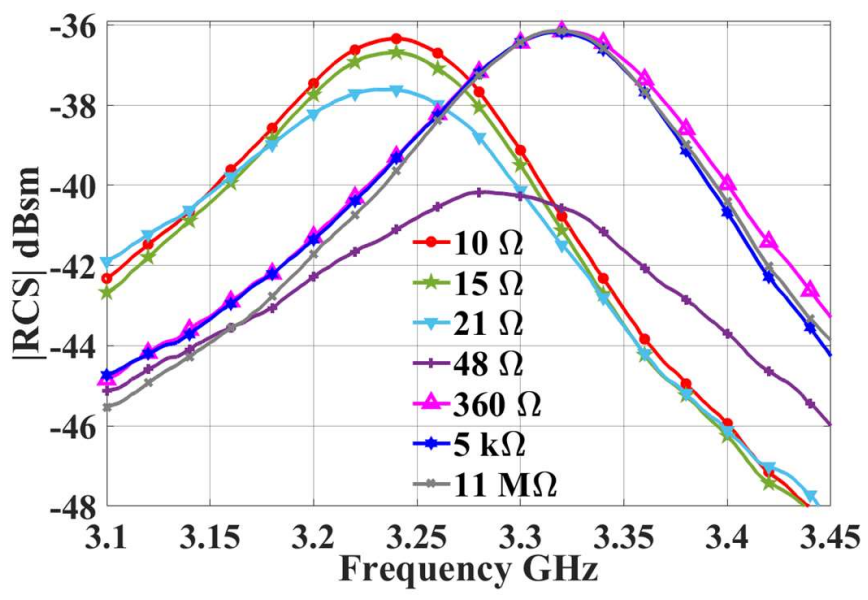

Fig. 18. Experimentally obtained RCS backscatter magnitude of electronically reconfigurable resonator presented in Fig. 17 for different impedance values of integrated CBRAM switch.

Classically, as this REP has just one integrated CBRAM switch, it could basically operate only in two states, i.e. ON (set) and OFF (reset) states. Here, one could observe that an impedance level lower than around $21 \Omega$ forces the REP to resonate in the so called $\mathrm{ON}$ state and impedance greater than $360 \Omega$ switches the resonance to OFF state. Furthermore, it is observed that among these ON and OFF states it is possible to 
define different CBRAM filament resistance values and hence represent different RCS magnitudes. These new RCS magnitude levels could be used to define new code states and effectively enhance coding capacity of tags utilizing these REPs. For example in this presented case the resonator could represent four states or 2 bits (which gives us two states in addition to normally proposed two states or 1 bit for this REP with one switch) considering the impedance values $10 \Omega, 15$ $\Omega, 21 \Omega$, and $>360 \Omega$. This represents a potentially new hybrid coding technique, combining frequency shift coding [6] and RCS magnitude level coding [39].

In addition, in aspects where necessary, an REP may be disabled to represent an absence (which could also be considered as a potential coded state). This could be achieved by tuning impedance of the REP to represent an RCS level below a certain preset threshold. Like the response of state with $48 \Omega$ given in Fig. 18, which has a lower quality factor resulting in difficulties to detect in comparison to other sharp peaks.

Further detailed investigations on this idea of hybrid coding based on impedance tuning of CBRAM switches is in progress with the authors. The above experiment and its outcome is given here as an immediate information of such a capability of proposed resonator, to the readers.

\section{CONCLUSION}

In this article we have presented the main aspects of design and realization of a fully printed fully passive electronically rewritable chipless RFID tag, utilizing solid state non-volatile CBRAM switching technology on PET substrates, through thermal transfer printing techniques. We have affirmatively introduced the following advances and novelties through this study in electronically rewritable chipless RFID technology.

- This is the first time such a printing process compatible with roll to roll printing technique is utilized to realize CBRAM RF switches, or an electronically rewritable chipless RFID tag using these switches.

- Proposed tags were realized on flexible PET laminates. Tags consist of three rewritable resonators (REP) each. And, one REP represents three coded states each. Fabricated tags were successfully programed and exhibit distinct readable resonant RCS backscatter response for all possible 27 switch combinations showing reliability of the design.

- We have presented and validated successfully an electrical model that precisely approximates behavior of the proposed chipless tag, and assuage the requirement of waiting for results of time-consuming multiple $3 \mathrm{D}$ full wave EM simulation studies, for all possible combinations defined by a given electronically rewritable chipless RFID tag of the proposed type.

- We have also presented the idea of quantization (or control) of RCS magnitude values of a rewritable resonator, by controlling the filament resistance values of integrated CBRAM switch. This experiment and its outcome trigger the conception of a possible novel hybrid encoding technique that could be used in future works.
Electronically rewritable chipless RFID tags presented herewith could find their potential application, as a superior replacement of optical barcodes. Moreover, such a technology of fabrication of electronically rewritable chipless RFID tags as presented herewith, is compatible with mass production in an industrial environment and could contribute to cheap and efficient smart identifiers of the future.

Future works related to these electronically rewritable chipless RFID tags, and the concept of fully printed reconfigurable $\mathrm{RF} /$ microwave devices based on CBRAM switching technology, includes detailed analysis of features such as maximum number of switching cycles, investigation of techniques for impedance control of CBRAM switches, miniaturization of chosen resonators, research on strategies to maximize RCS magnitude, studies on real-world environmental interactions of presented tags, and effects of orientations of tag with respect to reader system, and similar.

\section{REFERENCES}

[1] A. Vena, E. Perret, and S. Tedjini, Chipless RFID based on RF Encoding Particle: Realization, Coding and Reading System. New York, NY, USA: ISTE Press - Elsevier, 2016.

[2] N. C. Karmaker, "Tag, You're It Radar Cross Section of Chipless RFID Tags," IEEE Microw. Mag., vol. 17, no. 7, pp. 64-74, Jul. 2016, doi: 10.1109/MMM.2016.2549160.

[3] S. Tedjini, N. Karmakar, E. Perret, A. Vena, R. Koswatta, and R. EAzim, "Hold the Chips: Chipless Technology, an Alternative Technique for RFID,” IEEE Microw. Mag., vol. 14, no. 5, pp. 56-65, Jul. 2013, doi: 10.1109/MMM.2013.2259393.

[4] S. Preradovic and N. C. Karmakar, "Chipless RFID: Bar Code of the Future," IEEE Microw. Mag., vol. 11, no. 7, pp. 87-97, Dec. 2010, doi: 10.1109/MMM.2010.938571.

[5] E. Perret, Radio Frequency Identification and Sensors: From RFID to Chipless RFID. Hoboken, NJ, USA: Wiley, 2014.

[6] A. Vena, E. Perret, and S. Tedjini, "Chipless RFID Tag Using Hybrid Coding Technique," IEEE Trans. Microw. Theory Tech., vol. 59, no. 12, pp. 3356-3364, Dec. 2011, doi: 10.1109/TMTT.2011.2171001.

[7] R. S. Nair, E. Perret, S. Tedjini, and T. Baron, "A Group-Delay-Based Chipless RFID Humidity Tag Sensor Using Silicon Nanowires," IEEE Antennas Wirel. Propag. Lett., vol. 12, pp. 729-732, 2013, doi: 10.1109/LAWP.2013.2270929.

[8] I. Jalaly and I. D. Robertson, "Capacitively-tuned split microstrip resonators for RFID barcodes," in 2005 European Microwave Conference, Paris, France, Oct. 2005, vol. 2, doi: 10.1109/EUMC.2005.1610138.

[9] E. M. Amin, M. S. Bhuiyan, N. C. Karmakar, and B. Winther-Jensen, "Development of a Low Cost Printable Chipless RFID Humidity Sensor," IEEE Sens. J., vol. 14, no. 1, pp. 140-149, Jan. 2014, doi: 10.1109/JSEN.2013.2278560.

[10] M. Borgese, F. A. Dicandia, F. Costa, S. Genovesi, and G. Manara, "An Inkjet Printed Chipless RFID Sensor for Wireless Humidity Monitoring," IEEE Sens. J., vol. 17, no. 15, pp. 4699-4707, Aug. 2017, doi: 10.1109/JSEN.2017.2712190.

[11] J. M.P., A. Vena, B. Sorli, and E. Perret, "Solid-State ConductiveBridging Reconfigurable RF-Encoding Particle for Chipless RFID Applications," IEEE Microw. Wirel. Compon. Lett., vol. 28, no. 6, pp. 506-508, Jun. 2018, doi: 10.1109/LMWC.2018.2830702.

[12] A. A. C. Alves, D. H. Spadoti, and L. L. Bravo-Roger, "Optically Controlled Multiresonator for Passive Chipless Tag," IEEE Microw. Wirel. Compon. Lett., vol. 28, no. 6, pp. 467-469, Jun. 2018, doi: 10.1109/LMWC.2018.2824726

[13] Marco Garbati, Etienne Perret, Romain Siragusa, Chipless RFID Reader Design for Ultra-Wideband Technology - 1st Edition, 1st ed. ISTE Press - Elsevier, 2018.

[14] A. Vena, A. A. Babar, L. Sydanheimo, M. M. Tentzeris, and L. Ukkonen, "A Novel Near-Transparent ASK-Reconfigurable InkjetPrinted Chipless RFID Tag," IEEE Antennas Wirel. Propag. Lett., vol. 12, pp. 753-756, 2013, doi: 10.1109/LAWP.2013.2270932. 
[15] Md. A. Islam and N. C. Karmakar, "A Novel Compact Printable DualPolarized Chipless RFID System," IEEE Trans. Microw. Theory Tech., vol. 60, no. 7, pp. 2142-2151, Jul. 2012, doi: 10.1109/TMTT.2012.2195021.

[16] J. Methapettyparambu Purushothama, A. Vena, B. Sorli, and E. Perret, "Electronically Re-Configurable, Non-Volatile, Nano-Ionics-Based RF-Switch on Paper Substrate for Chipless RFID Applications,' Technologies, vol. 6, no. 3, p. 58, Jun. 2018, doi: 10.3390/technologies6030058.

[17] M. N. Kozicki and W. C. West, "Programmable metallization cell structure and method of making same," US5761115A, Jun. 02, 1998.

[18] Adesto Technologies, "RM24C32DS 32-Kbit 1.65V Minimum Nonvolatile Serial EEPROM I2C Bus." Adesto Technologies, 2016, Accessed: Jan. 09, 2019. [Online]. Available: https://www.adestotech.com/about-us/cbram/.

[19] Adesto Technologies, "Press Release | Adesto Technologies Wins DARPA Award to Develop Sub-Threshold Non-Volatile, Embedded CBRAM Memory." Nov. 2010, Accessed: Jul. 04, 2019. [Online]. Available: http://www.adestotech.com/news-detail/adestotechnologies-wins-darpa-award-to-develop-sub-threshold-nonvolatile-embedded-cbram-memory/.

[20] J. A. Nessel, R. Q. Lee, C. H. Mueller, M. N. Kozicki, Minghan Ren, and J. Morse, "A novel nanoionics-based switch for microwave applications," in 2008 IEEE MTT-S International Microwave Symposium Digest, Atlanta, GA, USA, Jun. 2008, pp. 1051-1054, doi: 10.1109/MWSYM.2008.4633016.

[21] J. Nessel and R. Lee, "Chalcogenide nanoionic-based radio frequency switch," US 7.923,715 B2, Apr. 12, 2011.

[22] S. Pi, M. Ghadiri-Sadrabadi, J. C. Bardin, and Q. Xia, "Nanoscale memristive radiofrequency switches," Nat. Commun., vol. 6, p. 7519 Jun. 2015, doi: 10.1038/ncomms8519.

[23] A. Vena, E. Perret, S. Tedjini, C. Vallée, P. Gonon, and C. Mannequin, "A fully passive RF switch based on nanometric conductive bridge," in 2012 IEEE/MTT-S International Microwave Symposium Digest, Montreal, QC, Canada, Jun. 2012, pp. 1-3, doi: 10.1109/MWSYM.2012.6258428.

[24] H. Pagnia and N. Sotnik, "Bistable switching in electroformed metalinsulator-metal devices," Phys. Status Solidi A, vol. 108, no. 1, pp. 11-65, Jul. 1988, doi: 10.1002/pssa.2211080102.

[25] M.-J. Song, K.-H. Kwon, and J.-G. Park, "Electro-Forming and Electro-Breaking of Nanoscale Ag Filaments for Conductive-Bridging Random-Access Memory Cell using Ag-Doped Polymer-Electrolyte between Pt Electrodes," Sci. Rep., vol. 7, no. 1, p. 3065, Jun. 2017 doi: 10.1038/s41598-017-02330-x

[26] J. Jian, H. Chang, A. Vena, and B. Sorli, "Study and design of resistive switching behaviors in PMMA-based conducting-bridge random-access memory (CBRAM) devices," Microsyst. Technol., vol. 23, no. 6, pp. 1719-1725, Jun. 2017, doi: 10.1007/s00542-015-27546.

[27] J. M.P., A. Vena, A. Meghit, B. Sorli, and E. Perret, "Nafion-Based Fully Passive Solid-State Conductive Bridging RF Switch," IEEE Microw. Wirel. Compon. Lett., vol. 27, no. 12, pp. 1104-1106, Dec. 2017, doi: 10.1109/LMWC.2017.2764741

[28] Y. Yang, P. Gao, S. Gaba, T. Chang, X. Pan, and W. Lu, "Observation of conducting filament growth in nanoscale resistive memories," Nat. Commun., vol. 3, p. 732, Mar. 2012, doi: 10.1038/ncomms 1737 .

[29] J. Methapettyparambu Purushothama, "New Generation of Electronically Reconfigurable Passive Microwave Devices Based on Non-volatile CBRAM Switching Technology," $\mathrm{PhD}$ Thesis, Université Grenoble Alpes, Valence, France, 2019.

[30] J. Methapettyparambu Purushothama, A. Vena, B. Sorli, and E. Perret, "Investigation of Integrated Solid State Nano-Ionic Metal-InsulatorMetal Switches for Electronically Reconfigurable Band Stop Filter Applications," IET Microw. Antennas Propag., vol. 13, no. 12, pp. 1963-1968, Oct. 2019, doi: 10.1049/iet-map.2019.0180.

[31] Sigma-Aldrich, Inc., "Nafion ${ }^{\circledR}$ perfluorinated resin solution." SigmaAldrich, Inc., 2019, Accessed: Mar. 21, 2019. [Online]. Available: https://www.sigmaaldrich.com/catalog/product/aldrich/274704.

[32] M. N. Kozicki and H. J. Barnaby, "Conductive bridging random access memory - materials, devices and applications," Semicond. Sci. Technol., vol. 31, no. 11, p. 113001, Oct. 2016, doi: 10.1088/0268$1242 / 31 / 11 / 113001$.

[33] A. Vena et al., "Design of Chipless RFID Tags Printed on Paper by Flexography," IEEE Trans. Antennas Propag., vol. 61, no. 12, pp. 5868-5877, Dec. 2013, doi: 10.1109/TAP.2013.2281742.
[34] P. Kalansuriya, N. C. Karmakar, and E. Viterbo, "On the Detection of Frequency-Spectra-Based Chipless RFID Using UWB Impulsed Interrogation," IEEE Trans. Microw. Theory Tech., vol. 60, no. 12, pp. 4187-4197, Dec. 2012, doi: 10.1109/TMTT.2012.2222920.

[35] A. Vena, E. Perret, and S. Tedjini, "Design rules for chipless RFID tags based on multiple scatterers," Ann. Telecommun. - Ann. Télécommunications, vol. 68, no. 7-8, pp. 361-374, Aug. 2013, doi: 10.1007/s12243-013-0358-7.

[36] Z. Lu, M. Lanagan, E. Manias, and D. D. Macdonald, "Two-Port Transmission Line Technique for Dielectric Property Characterization of Polymer Electrolyte Membranes," J. Phys. Chem. B, vol. 113, no. 41, pp. 13551-13559, Oct. 2009, doi: 10.1021/jp9057115.

[37] O. Rance, R. Siragusa, P. Lemaître-Auger, and E. Perret, "Contactless Characterization of Coplanar Stripline Discontinuities by RCS Measurement," IEEE Trans. Antennas Propag., vol. 65, no. 1, pp. 251-257, Jan. 2017, doi: 10.1109/TAP.2016.2618480

[38] "ZT600 Series Industrial Printers | Zebra," Zebra Technologies. https:/www.zebra.com/gb/en/products/printers/industrial/zt600series.html (accessed Feb. 13, 2020).

[39] O. Rance, R. Siragusa, P. Lemaître-Auger, and E. Perret, "Toward RCS Magnitude Level Coding for Chipless RFID," IEEE Trans. Microw. Theory Tech., vol. 64, no. 7, pp. 2315-2325, Jul. 2016, doi: 10.1109/TMTT.2016.2562625.

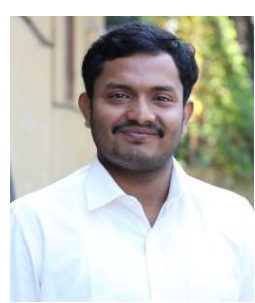

Jayakrishnan Methapettyparambu Purushothama received the B.Sc. and M.Sc. degree in Electronics and Electronics Science, respectively from the Mahatma Gandhi University, Kottayam, Kerala, India, and Cochin University of Science and Technology, Kochi, Kerala, India, subsequently in 2012 and 2014, with first rank position for both, and the Ph.D. degree in RF and Microwave Electronics Engineering from the Université Grenoble Alpes, France, in 2019.

From 2014 to 2016, he worked as a Research Fellow at the Centre for Research in Electromagnetics and Antennas, Department of Electronics, Cochin University of Science and Technology, Kochi, India. There he worked on implantable sensor antenna systems for wireless sensing, for medical applications. From 2016 to 2019, he worked as a Ph.D. scholar with the Laboratoire de Conception et d'Intégration des Systèmes (LCIS), Grenoble-INP, Université Grenoble Alpes, France, and since December 2019, he is working there as a Post-Doctoral Researcher in the ScattererID project (funded by the European Research Council (ERC) under European Union's Horizon 2020 research and innovation program), focused on development of CBRAM based RF switches, and their integration in passive $\mathrm{RF} /$ microwave devices, especially in chipless RFID applications. His research interests include chipless RFID, CBRAM based solid state RF switches, microwave based bio-sensors, implantable antennas, RCS studies, dielectric characterization, and similar.

Dr. Methapettyparambu Purushothama is a recipient of the $4^{\text {th }}$ prize and cash award in the $6^{\text {th }}$ international student paper competition organized by the International Union of Radio Science (URSI), held at URSI Atlantic Radio Science Meeting (AT-RASC) 2018, Gran Canaria, Spain, and also a finalist for student paper competition at the IEEE-MTT-S International Microwave Symposium (IMS) 2019, Boston, Massachusetts, USA. He has also received the Young Scientist Award (YSA) from URSI, at the URSI - General Assembly and Scientific Symposium (GASS) 2020, Rome, Italy. 


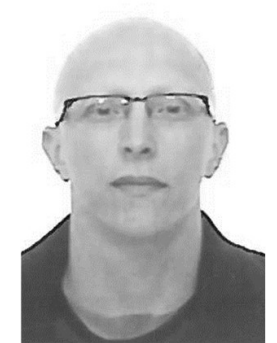

Sergio Lopez-Soriano was born in Barcelona, Spain. He received the bachelor's degree in Telecommunication Engineering from the Universitat Autònoma de Barcelona (UAB), Bellaterra, Spain, in 2012. He received the M.Sc. degree in Micro and Nanoelectronics Engineering and the Ph.D. degree in Electronics and Telecommunication Engineering from the Universitat Autònoma de Barcelona (UAB), Bellaterra, Spain, in 2013 and 2018 , respectively.

In 2018, he joined the Laboratoire de Conception et d'Intégration des Systemes (LCIS), Grenoble-INP, Université Grenoble Alpes, France, as a postdoctoral researcher in the ScattererID project (funded by the European Research Council (ERC) under European Union's Horizon 2020 research and innovation program), working on the analysis, characterization and programming of novel structures for implementing low cost switches, based on CBRAM cells, for radiofrequency devices.

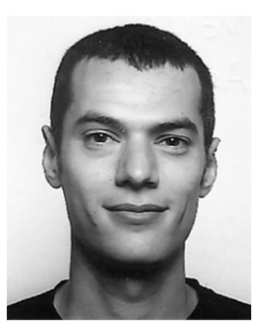

Arnaud Vena received the Eng. Dipl. degree in electrical engineering from the Institut National Polytechnique de Grenoble (Grenoble-INP), Grenoble, France, in 2005, and the $\mathrm{PhD}$ degree from the Université de Grenoble in 2012.

In 2005, he joined ACS Solution France SAS and was responsible for the development of RFID contactless card readers. In October 2009, he started his research within Grenoble-INP, mainly focused on the design of chipless RFID systems. From 20122013 he held a postdoctoral position at the Tampere University of Technology in Finland, in the field of conventional and chipless RFID sensors. Since September 2013, he is an Associate Professor at the Institut d'Electronique et des Systèmes (IES), University of Montpellier / CNRS, Montpellier, France. His current research interests are in the field of wireless sensors, RFID systems and printed electronics.

Brice Sorli received the M.S. degree in Applied Physics and $\mathrm{Ph} . \mathrm{D}$. degree in Electrical Engineering from Montpellier University, Montpellier, France, in 1998 and 2001, respectively. During this period, he worked on electronic measurements, instrumentation, thermal analysis and humidity sensors.

In 2002, he joined the "Electronic, Nanotechnologies \& Sensors Lab" (INL), Claude Bernard University, Lyon, France, where he has been involved in the design and implementation of nuclear magnetic resonance micro-probe for «Labs on chip» applications. Since 2005, he is an Associate Professor at the Institut d'Electronique et des Systèmes (IES), University of Montpellier / CNRS, Montpellier, France. His areas of interest include flexible sensors, printed electronics, and RFID applications.

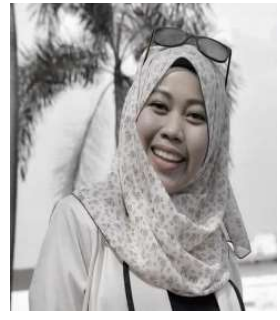

Ira Susanti was born in Padang, Indonesia, in 1990. She received the B.Ed degree in physics education from Padang State University, Indonesia, in 2012, and M.S. double degrees in material physics from Bandung Institute of Technology (ITB), Indonesia and in electrical engineering from Montpellier University, France, in 2016.

In 2015, she was an intern researcher at the Institute of Electronics and Systems (IES) Laboratory, Montpellier University, France. She worked on fabrication of Conductive Bridging Random Access Memory (CBRAM) and investigated the effects of Nafion as new material for solid electrolyte on the performances of resistive switching devices. Since 2016, she has been a physics lecturer with industrial engineering department, National Institute of Technology, Bandung, Indonesia.

She was a recipient of Directorate General of Higher Education Indonesia (DIKTI) scholarship in 2013 and French Government scholarship (BGF) in 2014.

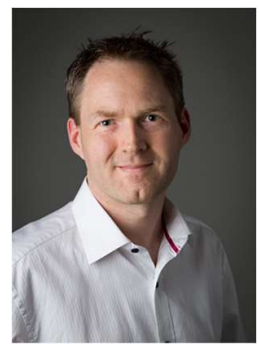

Etienne Perret (S'02-M'06-SM'13) received the Eng. Dipl. degree in electrical engineering from the Ecole Nationale Supérieure d'Electronique, d'Electrotechnique, d'Informatique, d'Hydraulique, et des Télécommunications, Toulouse, France, in 2002, and the M.Sc. and Ph.D. degrees in electrical engineering from the Toulouse Institute of Technology, Toulouse, in 2002 and 2005 , respectively.

From 2005 to 2006, he held a post-doctoral position with the Institute of Fundamental Electronics, Orsay, France. In 2006, he was appointed as an Associate Professor of electrical engineering at Grenoble INP - Institute of Engineering Univ. Grenoble Alpes, France. From 2014 to 2019, he has been a Junior Member with the Institut Universitaire de France, Paris, France, an institution that distinguishes professors for their research excellence, as evidenced by their international recognition. From 2015 to 2020, he has been an Appointed Member of the French National Council of Universities.

$\mathrm{He}$ has authored or co-authored over 200 technical conferences, letters and journal papers, and books and book chapters. He holds several patents. His works have generated about 2800 citations. His current research interests include electromagnetic modeling of passive devices for millimeter and submillimeter-wave applications, and wireless communications, especially RFID and chipless RFID, and also include advanced computer-aided design techniques based on the development of an automated co-design synthesis computational approach. Dr. Perret is a Technical Program Committee member of the IEEE International Conference on RFID. He was a recipient of the French Innovative Techniques for the Environment Award in 2013, the SEE/IEEE Leon Brillouin Award for his outstanding achievement in the identification of an object in an unknown environment using a chipless label or tag in 2016, and the IEEE MTT-S 2019 Outstanding Young Engineer Award. He was a Keynote 
Speaker and the Chairman of several international symposiums. He was named one of the MIT Technology Review's French Innovator's under 35 for his work on chipless RFID in 2013. Etienne Perret was awarded an ERC Consolidator Grant in 2017 for his project ScattererID, by the European Research Council (ERC) under European Union's Horizon 2020 research and innovation program. 